\title{
A review of large biochemistry analysers
}

John Fyffe,

Clinical Biochemistry, Queen Elizabeth II Hospital, Howlands, Welwyn Garden City, $A L 74 H Q$ UK

\section{P. Daga}

Chemical Pathology, Ashford Hospital, Ashford, Middlesex, UK

\section{and M. J. Roddis}

Chemical Pathology, Edgware General Hospital, Edgware, Middlesex HA 8 OAD, UK

\section{Introduction}

In recent years the numbers of large biochemistry analysers on the UK market has significantly increased and it was felt that a review of their specification in a comparative form may help prospective purchasing laboratories select from the large number of analysers available those that would be relevant for their purpose.

\section{Questionnaire}

To this end a questionnaire was sent out in January 1987 to 80 companies in the UK known to be involved in supplying biochemistry laboratories, requesting that a copy of the questionnaire be completed for each analyser they support on the UK market. The analyser was defined as being able to produce more than 100 test results for any of a group of the 23 most commonly performed biochemistry tests. Twenty-one companies replied positively, providing information for 69 analysers, which is presented in tables 1-16.

\section{Results}

Each table represents the manufacturer's answer to questions on one or more subjects listed at the head of each column. All information presented here has been checked with the manufacturer, however, it should be stressed that the information has come from manufacturers and its validity has not been checked by the authors.

This exercise was carried out earlier, Fyffe [1] in 1985 and an attempt has been made to correct as many anomalities as possible. It is important that the information presented be used as a guide to specifications of analysers and prospective purchasers should check all these details with the appropriate manufacturer before proceeding to purchase.

\section{Questionnaire}

1.1 Manufacturer and model.

1.2 When was this model first available in UK?

1.3 Which tests have available recommended methodologies?

1.4 How long do you expect this instrument to be available in the UK?

2.1 Which samples are acceptable?

2.2 What is the sample tray/carriage capacity?

2.3 What sample volumes are required to perform the stated groups of analytes? $\mathrm{U} \& \mathrm{E}=\mathrm{Na}, \mathrm{K}, \mathrm{Cl}$, Bic, Urea, Creat. LFT $=$ Bili, T Prot, Alb, ALP, +3 other Enzymes. BONE $=\mathrm{Ca}$, PO4, Alb, ALP. GARD $=3$ Enzymes. MISC = Cholesterol, Triglyceride, Urate. GLU = Glucose.

3.1 How many samples of each analyte group could be handled per hour, including calibration and QC? U\&E $=\mathrm{Na}, \mathrm{K}, \mathrm{Cl}, \mathrm{Bic}$, Urea, Creat. LFT $=$ Bili, T Prot, Alb, ALP, +3 other Enzymes. BONE $=\mathrm{Ca}, \mathrm{PO} 4, \mathrm{Alb}$, ALP. GARD $=3$ Enzymes. MISC $=$ Cholesterol, Triglyceride, Urate. GLU $=$ Glucose.

3.2 Are tests performed in batches?

3.3 What is the maximum batch size? (Where applicable.)

3.4 Does the instrument analyse samples selectively, in profiles or both?

3.5 What is the maximum number of samples/tests which can be analysed without operator intervention?

3.6 Has the instrument been the subject of a DHSS or other independent evaluation? If so, please quote.

3.7 What is the claimed within and between batch imprecision for each analyte.

4.1 What is the recommended frequency of calibration?

4.2 (a) Do you recommend aqueous or serum based calibrant?

4.2 (b) Can the instrument be calibrated by the operator using his existing assayed material?

4.2 (c) How many calibrators are required? Please state $\mathrm{max} / \mathrm{min}$. 
5.1 Can the instrument be safely used for handling high risk samples? (For example Hepatitis B, AIDS.) NB. In a normal clinical laboratory environment and using normal laboratory practices.

5.2 Is there a recommended method of decontamination in normal use and before planned maintenance?

5.3 Does the instrument comply with the ESCHLE code for electrical safety? (Ref: Health Equipment Information, No. 140, April 1985).

5.4 Please state any BSI standard with which the instrument complies.

6.1 Can the routine work be interrupted for stat samples?

6.2 How long would it take to produce a U\&E and Glucose result from $(a)$ Start up $(b)$ Standby? U\&E $=$ Na, $\mathrm{K}, \mathrm{Cl}$, Bic, Urea, Great, and glucose.

7.1 What services are required for installation?

7.2 Is the instrument floor or bench standing?

7.3 How much space is required including service access? (In m.)

7.4 What is the weight? (In kg.)

7.5 Are operator training courses available? If so $(a)$ how long, $(b)$ how many staff, $(c)$ what is the cost?

7.6 How many instruments are installed in the UK?

8.1 (a) What is the downtime per year for planned maintenance, not including daily maintenance?

(b) How long does daily maintenance take (for example of weekly divided by 5 etc.)?

8.2 Which types of service contract are available and at what cost?

8.3 Does the cost of service contract depend on the use of a specific manufacturer's reagents, spares or consumables?

8.4 How long is the warranty?

8.5 Does the warranty depend on the use of a specific manufacturer's reagents, spares or consumables?

8.6 How long do you expect to support maintenance of the instrument?

9.1 (a) In its standard form without any optional data manager, can the instrument $\log / \mathrm{monitor} / \mathrm{provide}$ statistics for quality control results?

(b) As above but with a data manager.

9.2 (a) In its standard form can the instrument hold patient identification and results?

(b) As above but with a data manager.

9.3 (a) In its standard form what is the storage capacity for QC/PID/RESULTS?

(b) As above but with a data manager.

9.4 (a) Is the standard instrument fitted with an RS 232/232 C compatible ONE way port?

(b) As above but with a TWO way port.

(c) If not, please quote price for $(a)$ and $(b)$.

9.5 (a) In its standard form is the report format fixed or user definable for printing results/PID on various sizes of paper?

(b) As above but with data manager.

9.6 (a) In its standard form is it possible to add results produced on other equipment to the report? (i) For on board chemistries (ii) For other chemistries produced on other instruments?

(b) As above but with a data manager.

9.7 (a) In its standard form can the instrument produce statistics required for the Korner report including listings for (i) Consultant, (ii) Locality, for example ward, and (iii) Clinical Specialty?

(b) As above but with a data manager.

9.8 (a) Is help available to link the analyser to an existing or future laboratory computer system?

(b) If so, what is the approx cost of (i) hardware (ii) software?

10.1 What is the basic price of the instrument? (In pounds sterling.)

10.2 (a) Cost of the ISE unit?

(b) Cost of data manager?

NB. State if included in 10.1 .

10.3 What is the expected recommended reagent cost to perform each group of analytes (see below) on 100 samples?

10.4 If 100 samples were analysed for each of the analyte groups (see below), what would be the cost of the consumables used in the analytical process?

10.5 (a) What is the volume of calibrant required to calibrate all on board chemistries once?

(b) What is the cost per $\mathrm{ml}$. of the recommended calibrant?

(c) What is the reconstituted volume and stability of the recommended calibrant?

$\mathrm{U} \& \mathrm{E}=\mathrm{Na}, \mathrm{K}, \mathrm{Cl}$, Bic, Urea, Creat. LFT = Bili, T Prot, Alb, ALP, + 3 other Enzymes. BONE = Ca, PO4, Alb, ALP. CARD = 3 Enzymes. MIS = Cholesterol, Triglyceride, Urate. GLU = Glucose. NB. for ALL, not all tests may be available at any one time. 


\begin{tabular}{|c|c|c|c|c|c|c|}
\hline 10 & 1.1 & 1.2 & 1.3 & & & 1.4 \\
\hline 1 & ABBOTT UP SUPER & 1979/83 & $19 / 21+20+E$ & max? & NO $\mathrm{Na} / \mathrm{K}$ & $1990+$ \\
\hline 2 & AMERICAN MONITOR PARALLEL & MID 1982 & $22 / 22+3$ & $\operatorname{MAX} 30$ & & $7-10 \gamma$ \\
\hline & AMERICAN RONITOR PERSPECTIVE & SEP 1985 & $22 / 22+8$ & $\max 64$ & & $7-10 \gamma$ \\
\hline 1 & BAKER EMCORE II & JAN 1985 & $19 / 22+7.4+E$ & & $\mathrm{NO} \mathrm{Na} / \mathrm{K}$ & $10 \mathrm{r}$ \\
\hline & BAKER SPIRIT & 1987 & $21 / 22$ & $\operatorname{MAX} 20$ & NO HBDH & INDEFF \\
\hline & BCL HITACHI 704 & 1986 & $22 / 22+21+E$ & & & $7 Y$ \\
\hline & BCL HITACHI 705 & 1981 & $21 / 21+14+E$ & & & RECONDIT \\
\hline 3 & BCL HITACHI 737 & 1985 & $21 / 21+16+E$ & & & $5 \gamma$ \\
\hline & BDH EPPENDORF EASY & JAN 1986 & $21 / 22+7+E$ & & & $310 \mathrm{r}$ \\
\hline 10 & BDH EPPENDORF EPOS & JAN 1985 & $20 / 22+19+E$ & & $\mathrm{NO} \mathrm{Na} / \mathrm{K}$ & s10r \\
\hline 11 & BOH EPPENDORF ERIS & MAY 1984 & $22 / 22+14$ & $\max 23$ & & $s 10 \mathrm{r}$ \\
\hline 12 & BDH EPPENDORF EXSEL & JUN 1985 & $22 / 22+19+E$ & $\max 24$ & & slor \\
\hline 13 & BDH EPPENDORF EXTRA & JUN 1985 & $22 / 22+14$ & $\max 43$ & & n10r \\
\hline 14 & BDH OLYYPUS AUS021 & MAY 1987 & $22 / 22+19+E$ & $\operatorname{MAX} 27$ & & slor \\
\hline 15 & BDH OLYMPUS AUS121 & MAY 1987 & $22 / 22+19+E$ & $E \max 19$ & & slor \\
\hline 16 & BDH OLYMPUS AU5031 & MAY 1987 & $22 / 22+19+E$ & MAX 27 & & s10Y \\
\hline 17 & BDH OLYMPUS AU5131 & MAY 1987 & $22 / 22+19+E$ & $E \max 14$ & & s10r \\
\hline 18 & BDH OLYMPUS AU504I & MAY 1987 & $22 / 22+19+E$ & $\operatorname{maX} 35$ & & $>10 Y$ \\
\hline 19 & BDH OLYMPUS AU5141 & MAY 1987 & $22 / 22+19+E$ & GAX 18 & & $>10 \mathrm{Y}$ \\
\hline 20 & BDH OLYMPUS AU5061 & MAY 1987 & $22 / 22+19+E$ & $\max 26$ & & $>10 Y$ \\
\hline 21 & BDH OLYMPUS AUSOBI & NAY 1987 & $22 / 22+19+E$ & $\sum \max 34$ & & $310 \mathrm{r}$ \\
\hline 22 & BECKMAN SYMCHRON AS4 & 1980 & $13 / 22$ & $\max 5$ & & $6 Y$ \\
\hline 23 & BECKMAN SYMCHRON ASB & 1981 & $13 / 22$ & $\max 9$ & & $6 Y$ \\
\hline 24 & BECKMAN SYMCHRON AS ENZYME & 1982 & $22 / 22$ & $\max 13$ & & $6 Y$ \\
\hline 25 & BECKHAN SYNCHRON AS LIPID & 1983 & $22 / 22$ & $\max 11$ & & $6 Y$ \\
\hline 26 & BECKKAN SYMCHRON AS MINI IDEAL & 1983 & $22 / 22$ & $\max 17$ & & $6 Y$ \\
\hline 27 & BECKHAN SYMCHRON AS IDEAL & 1983 & $22 / 22$ & $\max 23$ & & $6 y$ \\
\hline 28 & BECKMAN SYMCHRON CX3 & EARLY 87 & $22 / 22$ & $\max 8$ & & $10 \mathrm{r}$ \\
\hline$\angle Y$ & BECKMAN SYMCHRON CX4 & EARLY 88 & $22 / 22$ & $\operatorname{maX} 24$ & & $10 Y$ \\
\hline 30 & BECKMAN SYMCHRON CX5 & EARLY 88 & $22 / 22$ & $\max 28$ & & $10 \mathrm{Y}$ \\
\hline 31 & BECKHAN SYWCHROM CX7 & LATE 88 & $22 / 22$ & $\operatorname{MAX} 32$ & & $10 \mathrm{r}$ \\
\hline 32 & BIO AUTOMATED ASCA & 1987 & $19 / 22+51+E$ & & & IMDEFF \\
\hline 33 & CHEMLAB CHEHILYSER & SEP 1982 & $15 / 22$ & $\max 6$ & & n10Y \\
\hline 34 & CHEMLAB SYSTEM4 & 1983 & $15 / 22$ & $\max 9$ & & s10r \\
\hline 35 & CLINICON PRISHA II & 1986 & $22 / 22+7+E$ & $\operatorname{MaX} 48$ & & n10Y \\
\hline 36 & CIBA CORNING 580 ALLIANCE & MID 1987 & $22 / 22+2$ & & & $5 Y$ \\
\hline 37 & CIBA CORNING 570 & LATE 87 & $21 / 22+3$ & & & $5 Y$ \\
\hline 38 & CIBA CORNING EXPRESS & LATE1987 & $19 / 22$ & & $\mathrm{NO} \mathrm{Na} / \mathrm{K}$ & $5 Y$ \\
\hline 39 & COULTER CPA & MAR 1987 & $19 / 22+4$ & MAX 8 & & INDEFF \\
\hline 40 & COULTER DACOS 2 & AU6 1983 & $22 / 22+6$ & $\max 30$ & & FUR NOT \\
\hline 41 & DUPONT DIMENSION 380 & HID 1986 & $19 / 22+18$ & $\operatorname{maX} 37$ & & UPTO1OY \\
\hline 42 & DUPONT DIMENSION 760 & HID 1986 & $19 / 22+18$ & $\max 37$ & & UPTOLOY \\
\hline 43 & GREINER 6-400 & 1983 & $22 / 22+6$ & $\max 30$ & & $>5 Y$ \\
\hline 14 & GREIMER 6-450 & 1986 & $22 / 22+9$ & $\operatorname{maX} 30$ & & >10Y \\
\hline 45 & GREIMER TANDEN 2 & 1986 & $22 / 22+39$ & $\max 60$ & & nor \\
\hline 16 & GREINER TANDEM 3 & 1986 & $22 / 22+39$ & $\max 90$ & & sior \\
\hline 47 & IL GENESIS 21 & FEB 1985 & $22 / 22+5+E$ & $\max 21$ & & $7-10 \mathrm{r}$ \\
\hline $48-5$ & IL MOMARCH & SEP 1985 & $22 / 22+20+E$ & $\max 23$ & & $7-10 Y$ \\
\hline 49 & IL MOMARCH PLUS 2 & 1985 & $22 / 22+20+E$ & $5 \max 23$ & & $7-10 r$ \\
\hline 50 & KODAK EKTACHEN DT & 1985 & $18 / 21+4$ & & & INDEFF \\
\hline 51 & KODAK EKTACHEN 700 & 1987 & $20 / 21+10$ & & & INDEFF \\
\hline 52 & KONE PROGRESS & MAR 1984 & $22 / 22+30+E$ & $=\max 27$ & & $75 \gamma$ \\
\hline 3 & KOME SPECIFIC & SEP 1986 & $22 / 22+30$ & & & $25 \%$ \\
\hline & NOVA BIOHED $1+1$ & 1977 & Na/K ONLY & & & $5 Y$ \\
\hline & NOVA BIOHED $4+4$ & 1981 & $\mathrm{Na} / \mathrm{K} / \mathrm{Cl} / \mathrm{BiC}$ & ic ONLY & & $5 \gamma$ \\
\hline 10 & MOVA BIOHED $5+5$ & 1982 & $\mathrm{Na} / \mathrm{K} / \mathrm{Cl} \mathrm{ONL}$ & & & $5 Y$ \\
\hline 57 & ROCHE COBAS FARA & 1984 & $22 / 22+10+E$ & & & $5 Y$ \\
\hline 58 & ROCHE COBAS MIRA & 1984 & $22 / 22+10+E$ & & & $7 Y$ \\
\hline 59 & TECHNICON ASSIST & 1987 & $17 / 22$ & $\operatorname{MAX} 12$ & $2 \mathrm{NO} \mathrm{Na} / \mathrm{K}$ & $27 \mathrm{Y}$ \\
\hline & TECHNICON CHEN 1 & JUN 1985 & $22 / 22+3$ & $\max 35$ & & $37 \%$ \\
\hline & TECHNICON RA-1000 & 1983 & $22 / 22+80+E$ & $=\max 15$ & & $27 Y$ \\
\hline & TECHWICON RA-2X & 1984 & $22 / 22+80+E$ & $\max 27$ & & 274 \\
\hline & TECHNICON RA-500 & 1985 & $20 / 22+80+E$ & $\max 12$ & $2 \mathrm{NO} \mathrm{Ma} / \mathrm{K}$ & $27 \mathrm{Y}$ \\
\hline & TECHWICON RA-XT & 1986 & $22 / 22+80+E$ & $\max 26$ & & 274 \\
\hline & TECHWICON SUAC II & 1981 & $21 / 22+2$ & $\max 23$ & & $27 Y$ \\
\hline & TECHWICON SRA 2000 & 1983 & $22 / 22+80+E$ & $\max 35$ & & $37 Y$ \\
\hline & ULTROLAB ENI GEN PROFILER & JAN 1987 & $20 / 22$ & & $\mathrm{NO} \mathrm{Ma} / \mathrm{K}$ & s10r \\
\hline & ULTROLAB ULTROCHEH I & JUL 1987 & M/DA & & & $N / D A$ \\
\hline & VITAL SCIENTIFIC VITALA & JUN 1987 & $19 / 22+3+E$ & $\max 40$ & & zlor \\
\hline
\end{tabular}




\begin{tabular}{|c|c|c|c|c|c|c|c|c|c|c|c|}
\hline No & IMST & 2.1 & 2.2 & 2.3 U/E & 2.3 LFT & 2.3 BONE & 2.3 CARD & $2.3 \mathrm{HISC}$ & 2.3 GLU & $2.3 \mathrm{ALL}$ & \\
\hline 1 & ABBOTT UPS & PSU & 32 & NO U/E & 44 & 24 & 18 & 18 & 5 & 104 & NO U/E \\
\hline 2 & PARALLEL & PSU & 150 & 75 & 135 & 45 & 128 & 11 & 4 & 394 & \\
\hline 3 & PERSPECTIVE & PSU & 40 & 98 & 84 & 30 & 59 & 8 & 2 & 281 & \\
\hline 4 & ENCORE II & PSU & 96 & NO U/E & 59 & 19 & 33 & 15 & $*$ & 126 & NO U/E \\
\hline 5 & SPIRIT & PSU & 40 & 161 & 110 & 27 & 61 & 27 & 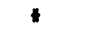 & 386 & \\
\hline 6 & HITACHI 704 & PSUMB & 50 & 49 & 68 & 33 & 40 & 25 & 15 & 152 & \\
\hline 7 & HITACHI 705 & PSUNB & 48 & 119 & 58 & 23 & 30 & 15 & 5 & 222 & \\
\hline 8 & HITACHI 737 & PSUMB & 60 & 37 & 44 & 18 & 29 & 12 & 6 & 109 & \\
\hline 9 & EASY & PSU & 9 & 120 & 230 & $N / D A$ & 120 & N/DA & 5 & N/DA & \\
\hline 10 & EPOS & PSU & 200 & NO U/E & 88 & 22 & 45 & 10 & 5 & 170 & NO U/E \\
\hline 11 & ERIS & PSU & 100 & 84 & 91 & 22 & 60 & 17 & 5 & 279 & \\
\hline 12 & EXSEL & PSU & 300 & 84 & 88 & 22 & 45 & 10 & 5 & 254 & \\
\hline 13 & EXTRA & PSU & 200 & 84 & 91 & 22 & 60 & 17 & 5 & 274 & \\
\hline 14 & AUS021 & PSU & 300 & 81 & 73 & 22 & 45 & 10 & 5 & 239 & \\
\hline 15 & AU5121 & PSU & 300 & 81 & 73 & 22 & 45 & 10 & 5 & 239 & \\
\hline 16 & AU5031 & PSU & 300 & 81 & 73 & 22 & 45 & 10 & 5 & 239 & \\
\hline 17 & AU5131 & PSU & 300 & 81 & 73 & 22 & 45 & 10 & 5 & 239 & \\
\hline 18 & AU5041 & PSU & 300 & 81 & 73 & 22 & 45 & 10 & 5 & 239 & \\
\hline 19 & AU5141 & PSU & 300 & 81 & 73 & 22 & 15 & 10 & 5 & 239 & \\
\hline 20 & AU5061 & PSU & 300 & 81 & 73 & 22 & 45 & 10 & 5 & 239 & \\
\hline 21 & AU5081 & PSU & 300 & 81 & 73 & 22 & 45 & 10 & 5 & 239 & \\
\hline 22 & AS4 & PSU & 38 & 110 & $\star$ & $*$ & $*$ & 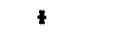 & 10 & $\$$ & \\
\hline 23 & AS8 & PSU & 38 & 110 & 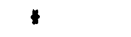 & 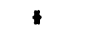 & t & $\star$ & 10 & t & \\
\hline 24 & AS ENZYME & PSU & 38 & 110 & 144 & 48 & 85 & $*$ & 10 & $*$ & \\
\hline 25 & AS LIPID & PSU & 38 & 110 & 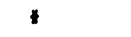 & $\star$ & $\star$ & 30 & 10 & $*$ & \\
\hline 26 & HINI IDEAL & PSU & 38 & 110 & 144 & 48 & 85 & 30 & 10 & $*$ & \\
\hline 27 & AS IDEAL & PSU & 38 & 110 & 144 & 48 & 85 & 30 & 10 & 427 & \\
\hline 28 & $\mathrm{c} \times 3$ & PSU & 80 & 112 & $*$ & $\star$ & $*$ & $*$ & 10 & $*$ & \\
\hline 29 & $C X_{4}$ & PSU & 80 & $N / D A$ & $N / D A$ & N/DA & $N / D A$ & N/DA & N/DA & N/DA & \\
\hline 30 & Cxs & PSU & 80 & N/DA & $N / D A$ & N/DA & $\mathrm{N} / \mathrm{DA}$ & N/DA & N/DA & $N / D A$ & \\
\hline 31 & $c \times 7$ & PSU & 80 & $N / D A$ & N/DA & N/DA & N/DA & N/DA & N/DA & N/DA & \\
\hline 32 & ASCA & PSU & 48 & 20 & 80 & 45 & 40 & 45 & $*$ & 230 & NO U/E \\
\hline 33 & CHEHILYSER & PSU & 80 & • & 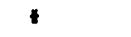 & 1 & $\star$ & $\star$ & * & 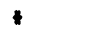 & \\
\hline 34 & SYSTEMA & PSU & 300 & $*$ & $*$ & $*$ & $\star$ & $\star$ & $*$ & $*$ & \\
\hline 35 & PRISHA II & PSU & 200 & 300 & 1000 & 200 & 300 & 200 & 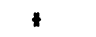 & 2000 & \\
\hline 36 & CORNING 580 & PSU & 90 & 135 & 235 & 145 & 185 & 130 & 117 & 325 & \\
\hline 37 & CORNING 570 & PSU & 90 & N/DA & N/DA & N/DA & N/DA & $M / D A$ & $N / D A$ & N/DA & \\
\hline 38 & CORNING EXPRESS & PSU & 40 & $N / D A$ & $M / D A$ & N/DA & $N / D A$ & N/DA & N/DA & $N / D A$ & \\
\hline 39 & CPA & PSU & 32 & NO U/E & 97 & 23 & 48 & 16 & 4 & 188 & NO U/E \\
\hline 40 & DACOS 2 & PSU & 80 & 150 & 48 & 19 & 14 & 10 & 3 & 278 & \\
\hline $4 !$ & DIMEMSION 380 & PSU & 60 & 128 & 156 & 17 & 68 & 23 & 3 & + & \\
\hline 42 & DIMENSION 760 & PSU & 120 & 128 & 156 & 17 & 68 & 23 & 3 & $*$ & \\
\hline 43 & GREIMER 6-400 & PSU & 30 & 145 & 125 & 35 & 60 & 30 & 5 & 325 & \\
\hline 44 & GREIMER G-450 & PSU & 30 & 145 & 125 & 35 & 60 & 30 & 5 & 325 & \\
\hline 45 & TAMDEN 2 & PSU & 60 & 145 & 125 & 35 & 60 & 30 & 5 & 325 & \\
\hline 46 & TANDEM 3 & PSU & 90 & 145 & 125 & 35 & 60 & 30 & 5 & 325 & \\
\hline 47 & GEMESIS 21 & PSU & 50 & 82 & 132 & 30 & 45 & 19 & 5 & 271 & \\
\hline 48 & MONARCH & PSU & 38 & 48 & 70 & 20 & 20 & 11 & 3 & 157 & \\
\hline 49 & MOMARCH PLUS 2 & PSU & 76 & 48 & 70 & 20 & 20 & 11 & 3 & 157 & \\
\hline 50 & EXTACHEN DT & PS & N/AP & 70 & 6ONO ALB & $N / A$ & 30 & 30 & 10 & $*$ & \\
\hline 51 & EXTACHEN 700 & PSU & 40 & 60 & 70 & 41 & 33 & 30 & 10 & 284 & \\
\hline 52 & PROGRESS & PSU & 84 & 145 & 31 & 8 & 20 & 10 & 2 & 214 & \\
\hline 53 & SPECIFIC & PSU & 84 & 145 & 31 & 8 & 20 & 10 & 2 & 214 & \\
\hline 54 & NOVA BIOHED $1+1$ & PSUMB & 40 & $*$ & 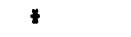 & $*$ & $\$$ & $\star$ & $*$ & $\$$ & \\
\hline 55 & NOVA BIOMED $4+4$ & PSUMB & 40 & 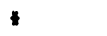 & $\star$ & - & $\star$ & $*$ & 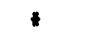 & + & \\
\hline 56 & MOVA BIOMED $5+5$ & PSUNB & 40 & $*$ & 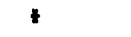 & $*$ & $*$ & $\star$ & $*$ & $*$ & \\
\hline 57 & COBAS FARA & PSU & 150 & 105 & 78 & 17 & 28 & 15 & $\star$ & 243 & \\
\hline 58 & COBAS MIRA & PSU & 90 & 105 & 108 & 17 & 43 & 15 & * & 289 & \\
\hline 59 & ASSIST & PSU & 16 & NO U/E & 90 & 30 & 50 & 35 & 5 & 240 & NO U/E \\
\hline 60 & CHEM 1 & PSU & 288 & 13 & 7 & 4 & 3 & 3 & 1 & 30 & \\
\hline 61 & $R A-1000$ & PSU & 30 & 35 & 90 & 30 & 50 & 35 & 5 & 240 & \\
\hline 62 & $R A-2 x$ & PSU & 60 & 35 & 90 & 30 & 50 & 35 & 5 & 240 & \\
\hline 63 & $R A-500$ & PSU & 30 & NO U/E & 90 & 30 & 50 & 35 & 5 & 240 & NO U/E \\
\hline 64 & $R A-X T$ & PSU & 30 & 35 & 90 & 30 & so & 35 & 5 & 240 & \\
\hline 65 & $\operatorname{snac}$ II & PS & 144 & 450 & 450 & 450 & 450 & 450 & 450 & 450 & \\
\hline 66 & SRA 2000 & PSU & 174 & 35 & 90 & 30 & so & 35 & 5 & 240 & \\
\hline 67 & GEM PROFILER & SPU & 45 & WO U/E & PROG & PROG & PROG & PRO6 & PROG & PROG & \\
\hline 68 & ULTROCHEN 1 & N/DA & 100 & NO U/E & 100 & 35 & 60 & 20 & $*$ & + & \\
\hline 69 & VITALAB 200 & PSU & 12 & N/DA & $M / D A$ & N/DA & M/DA & $N / D A$ & N/DA & N/DA & \\
\hline
\end{tabular}




\begin{tabular}{|c|c|c|c|c|c|c|c|}
\hline No & INST & 3.1 U/E & 3.1 LFT & 3.1 BONE & 3.1 CARD & 3.1 MISC & $3.16 \mathrm{LL}$ \\
\hline 1 & ABBOTT UPS & NO U/E & 44 & 84 & 93 & 77 & 465 \\
\hline 2 & PARALLEL & 240 & 240 & 240 & 240 & 240 & 240 \\
\hline 3 & PERSPECTIVE & 200 & 100 & 200 & 300 & 330 & 500 \\
\hline 4 & ENCORE II & NO U/E & 57 & 100 & 133 & 100 & N/DA \\
\hline 5 & SPIRIT & 40 & 30 & 60 & 40 & 60 & $N / D A$ \\
\hline 6 & HITACHI 704 & 60 & 30 & 45 & 90 & 60 & 180 \\
\hline 7 & HITACHI 705 & 60 & 30 & 45 & 90 & 60 & 180 \\
\hline 8 & HITACHI 737 & 75 & 150 & 300 & 300 & 300 & 300 \\
\hline 9 & EASY & 15 & 8 & 15 & 20 & 20 & 50 \\
\hline 10 & EPOS & NO U/E & 39 & 75 & 100 & 68 & 300 \\
\hline 11 & ERIS & 100 & 59 & 100 & 133 & 133 & 400 \\
\hline 12 & EXSEL & 100 & 98 & 175 & 233 & 201 & 700 \\
\hline 13 & EXTRA & 200 & 118 & 200 & 266 & 266 & 800 \\
\hline 14 & AU5021 & 100 & 100 & 100 & 100 & 100 & 100 \\
\hline 15 & AU5121 & 150 & 150 & 150 & 150 & 150 & 150 \\
\hline 16 & AU5031 & 150 & 150 & 150 & 150 & 150 & 150 \\
\hline 17 & AUs131 & 300 & 300 & 300 & 300 & 300 & 300 \\
\hline 18 & AU5041 & 150 & 150 & 150 & 150 & 150 & 150 \\
\hline 19 & Aus5141 & 300 & 300 & 300 & 300 & 300 & 300 \\
\hline 20 & AU5061 & 300 & 300 & 300 & 300 & 300 & 300 \\
\hline 21 & AU5081 & 300 & 300 & 300 & 300 & 300 & 300 \\
\hline 22 & AS4 & 65 & N/DA & N/DA & N/DA & N/DA & 65 \\
\hline 23 & A58 & 65 & N/DA & N/DA & N/DA & N/DA & 65 \\
\hline 24 & AS ENZYME & 65 & 50 & 50 & so & $N / D A$ & 65 \\
\hline 25 & AS LIPID & 65 & N/DA & N/DA & N/DA & 50 & 65 \\
\hline 26 & HINI IDEAL & 65 & 50 & 50 & 50 & 50 & 65 \\
\hline 27 & AS IDEAL & 65 & 50 & 50 & 50 & 50 & 65 \\
\hline 28 & $\mathrm{Cx} 3$ & 75 & $M / D A$ & $N / D A$ & N/DA & $M / D A$ & 75 \\
\hline 29 & $c \times 4$ & NO U/E & N/DA & N/DA & N/DA & N/DA & $N / D A$ \\
\hline 30 & $c \times 5$ & 75 & N/DA & N/DA & $M / D A$ & $M / D A$ & $N / D A$ \\
\hline 31 & $c \times 7$ & 75 & N/DA & N/DA & $N / D A$ & N/DA & N/DA \\
\hline 32 & ASCA & NO U/E & 30 & 55 & 73 & 55 & $N / D A$ \\
\hline 33 & CHEMILYSER & 120 & N/DA & 120 & N/DA & N/DA & 80 \\
\hline 34 & SYSTEMA & 80 & $M / D A$ & N/DA & N/DA & $M / D A$ & 80 \\
\hline 35 & PRISMA II & 300 & 300 & 300 & 300 & 300 & 300 \\
\hline 36 & CORNIME 580 & 100 & 100 & 100 & 100 & 100 & 100 \\
\hline 37 & CORNING 570 & 70 & 60 & 105 & 100 & 100 & 300 \\
\hline 38 & CORNING EXPRESS & NO U/E & 26 & 45 & 60 & 60 & 180 \\
\hline 39 & CPA & NO U/E & 22 & 33 & 60 & 60 & 220 \\
\hline 40 & DACOS 2 & 45 & 64 & 112 & 150 & 150 & 450 \\
\hline 41 & DIMENSION 380 & 48 & 25 & 35 & 10 & 45 & 170 \\
\hline 42 & DIMENSION 760 & 95 & 50 & 70 & 80 & 90 & 340 \\
\hline 43 & GREINER 6-400 & 42 & 37 & 75 & 100 & 100 & 300 \\
\hline 44 & GREIMER G-450 & 57 & 50 & 100 & 133 & 133 & 400 \\
\hline 15 & TANDEY 2 & 114 & 100 & 200 & 266 & 266 & 800 \\
\hline 46 & TAMDEN 3 & 170 & 150 & 300 & 400 & 400 & 1200 \\
\hline 47 & GEMESIS 21 & 33 & 25 & 45 & 60 & 60 & 200 \\
\hline 48 & MONARCH & 60 & 35 & 100 & 140 & 135 & 250 \\
\hline 49 & MOMARCH PLUS 2 & 120 & 110 & 200 & 280 & 270 & 500 \\
\hline 50 & EXTACHEM DT 7 & 7 & 6 & $N / D A$ & 8 & 21 & 65 \\
\hline 51 & EKTACHEN 700 & 90 & 71 & 90 & 120 & 120 & 360 \\
\hline 52 & PROBRESB & 40 & 26 & 45 & 60 & 60 & N/DA \\
\hline 53 & SPECIFIC & NO U/E & 20 & 33 & 43 & 43 & N/DA \\
\hline 54 & NOVA BIOMED $1+1$ & $*$ & 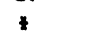 & 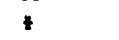 & 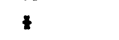 & 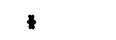 & $*$ \\
\hline 55 & NOVA BIOHED $4+4$ & 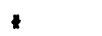 & $\star$ & + & 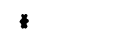 & $\star$ & + \\
\hline 56 & NOVA BIONED $5+5$ & : & $\star$ & $\star$ & 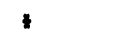 & $\star$ & + \\
\hline 57 & COBAS FARA & 36 & 28 & 56 & 70 & 67 & N/DA \\
\hline 58 & COBAS MIRA & 18 & 19 & 22 & 13 & 44 & $N / D A$ \\
\hline 59 & ABSIST & NO U/E & 10 & 15 & 20 & 20 & 60 \\
\hline 60 & CHEN 1 & 240 & 103 & 180 & 240 & 240 & 720 \\
\hline 61 & $R A-1000$ & 60 & 34 & 60 & 80 & 80 & 240 \\
\hline 62 & $R A-2 X$ & 120 & 69 & 120 & 160 & 160 & 480 \\
\hline 63 & $R A-500$ & NO U/E & 34 & 60 & 80 & 80 & 240 \\
\hline 64 & $R A-X T$ & 60 & 34 & 60 & 80 & 80 & 240 \\
\hline 65 & SMac II & 150 & 150 & 150 & 150 & 150 & 150 \\
\hline 66 & SRA 2000 & 150 & 150 & 150 & 150 & 150 & 150 \\
\hline 67 & GEA PROFILER & NO U/E & M/DA & M/DA & N/DA & M/DA & $N / D A$ \\
\hline 68 & ULTROLAB I & NO U/E & 20 & 35 & 40 & 50 & 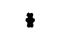 \\
\hline 69 & VITALAB 200 & NO U/E & 10 & 18 & 24 & 24 & 70 \\
\hline
\end{tabular}




\begin{tabular}{|c|c|c|c|c|c|c|}
\hline No & INBT & 3.2 & 3.3 & 3.4 & 3.5 & 3.6 \\
\hline 1 & ABBBOTT UPS & YES & 28 & EEL & 321 & YES QUOTED \\
\hline 2 & PARALLEL & Mo & & ВОтн & $150 /$ & YES MOT YET PUB \\
\hline 3 & PERSPECTIVE & CAN BE & 40 & BOTH & $40 / 1240$ & MOT YET PUBLISH \\
\hline 4 & ENCORE II & YES & 30 & BOTH & 281 & MOT YET PUBLISH \\
\hline 5 & SPIRIT & YES & 10 & BOTH & $40 / 640$ & No \\
\hline 6 & HITACHI 704 & No & N/APP & BOTH & $50 / 1300$ & TRIALS IM EUROP \\
\hline 7 & HITACHI 705 & No & M/APP & BOTH & $48 / 1248$ & DHSS \\
\hline 8 & HITACHI 737 & No & N/APP & BOTH & $60 / 1380$ & DH5S \\
\hline 9 & EASY & No & & PROF & $9 / 99$ & No \\
\hline 10 & EPOS & YES & 200 & SEL & $200 / 200$ & no \\
\hline 11 & ERIS & No & & BOTH & $100 / 100$ & YES \\
\hline 12 & EXSEL & CAN BE & 200 & BOTH & $300 / 1700$ & no \\
\hline 13 & EXTRA & No & & ВОTH & $200 / 3000$ & No \\
\hline 14 & AU5021 & No & & BOTH & $300 / 8100$ & NO \\
\hline 15 & AU5121 & NO & & BOTH & $300 / 5700$ & no \\
\hline 16 & AU5031 & NO & & BOTH & $300 / 8100$ & No \\
\hline 17 & A45131 & No & & BOTH & $300 / 4200$ & No \\
\hline 18 & AUS041 & No & & BOTH & $300 / 10500$ & No \\
\hline 19 & Aus141 & No & & BOTH & $300 / 5400$ & YES \\
\hline 20 & AU5061 & NO & & BOTH & $300 / 7800$ & No \\
\hline 21 & AU5081 & No & & ВОTH & $300 / 10200$ & no \\
\hline 22 & AS4 & No & & BOTH & $38 / 190$ & YES QUOTED \\
\hline 23 & A58 & No & & BOTH & $38 / 342$ & YES QUOTED \\
\hline 24 & AS ENZYHE & No & & BOTH & $38 / 494$ & YES QUOTED \\
\hline 25 & AS LIPID & No & & BOTH & $38 / 418$ & YES QUOTED \\
\hline 26 & MINI IDEAL & No & & BOTH & $38 / 646$ & YES QUOTED \\
\hline 27 & AS IDEAL & No & & BOTH & $38 / 874$ & YES QUOTED \\
\hline 28 & $c \times 3$ & No & & BOTH & $80 / 640$ & No \\
\hline 29 & $c \times 4$ & No & & BOTH & $80 / 1920$ & NO \\
\hline 30 & CX5 & No & & BOTH & $80 / 2240$ & No \\
\hline 31 & $\mathrm{CX7}$ & NO & & ВОTH & $80 / 2560$ & No \\
\hline 32 & ASCA & CAN BE & $38 / 1000$ & BOTH & 381 & No \\
\hline 33 & CHEMILYSER & YES & 480 & PROF & $80 /$ & YES QUOTED \\
\hline 34 & SYSTENA & YES & 300 & PROF & 3001 & NOT CLINICAL \\
\hline 35 & PRISMA II & CAN BE & & BOTH & $220 / 7040$ & NOT YET PUBLISH \\
\hline 36 & CORMING 580 & No & N/A & BOTH & $90 / 1980$ & No \\
\hline 37 & CORWING 570 & No & N/A & ВОTH & $90 / 2161$ & No \\
\hline 38 & CORWING EXPRESS & No & N/A & BOTH & $40 / 520$ & No \\
\hline 39 & CPA & YES & 64 & ВОТн & $64 / 64$ & No \\
\hline 40 & DACOS 2 & No & & BOTH & $80 / 2400$ & YES QUOTED \\
\hline 41 & DIMENSION 380 & CAN BE & 55 & BOTH & $55 / 55$ & NOT YET PUBLISH \\
\hline 42 & DIMENSION 760 & CAN BE & 55 & BOTH & $55 / 110$ & MOT YET PUBLISH \\
\hline 43 & GREIMER 6-400 & CAN BE & NO LIMIT & BOTH & $30 / 900$ & YES QUOTED \\
\hline 44 & GREIMER 6-450 & CAN BE & MO LIMIT & BOTH & $30 / 900$ & YES QUOTED \\
\hline 45 & TAMDEM 2 & CAN BE & NO LIMIT & BOTH & $60 / 1800$ & YES QUOTED \\
\hline 46 & TANDEM 3 & CAN BE & NO LIMIT & BOTH & $90 / 2700$ & YES QUOTED \\
\hline 47 & GENESIS 21 & & & BOTH & $50 / 1050$ & YES QUOTED \\
\hline 48 & MOMARCH & CAN BE & 38 & BOTH & $38 / 722$ & YES \\
\hline 49 & MONARCH PLUS 2 & CAM BE & 38 & BOTH & $76 / 1444$ & YES \\
\hline 50 & EKTACHEN DT & No & & SEL & $1 / 1$ & MOT YET PUBLISH \\
\hline 51 & EKTACHEN 700 & No & & SEL & $40 / 1200$ & No \\
\hline 52 & PROGRESS & CAM BE & NO LIMIT & BOTH & 1180 & YES QUOTED \\
\hline 53 & SPECIFIC & YES & NO LIMIT & BOTH & 1180 & No \\
\hline 54 & NOVA BIOMED $1+1$ & YES & 40 & PROF & $40 /$ & No \\
\hline 55 & MOVA BIOMED $4+4$ & YES & 40 & PROF & $40 /$ & NO \\
\hline 56 & MOVA BIOMED $5+5$ & YES & 40 & PROF & 401 & No \\
\hline 57 & COBAS FARA & YES & 28 & PROF & 128 & No \\
\hline 58 & COBAS MIRA & CAN BE & $90 / 72$ & BOTH & 172 & MO \\
\hline 59 & ASSIST & No & & SEL & $16 / 16$ & No \\
\hline 60 & CHEN 1 & No & No LIMIT & BOTH & 2881 & No \\
\hline 61 & $R A-1000$ & NO & & BOTH & $30 / 100$ & YES QUOTED \\
\hline 62 & $R A-2 X$ & No & & BOTH & $30 / 100$ & YES QUOTED \\
\hline 63 & $R A-500$ & No & & BOTH & $30 / 100$ & YES QUOTED \\
\hline 64 & $R A-X T$ & No & & BOTH & $30 / 100$ & YES QUOTED \\
\hline 65 & SMAC II & YES & NO LIMIT & PROF & $144 /$ & No \\
\hline 66 & SRA 2000 & YES & NO LIMIT & BOTH & $144 /$ & No \\
\hline 67 & GEM PROFILER & BOTH & UMLIMITED & BOTH & 201 & No \\
\hline 68 & ULTROLAB I & CAN BE & 100 & & 11500 & YES NOT YET PUB \\
\hline 69 & VITALAB 200 & & & BOIH & 127 & \\
\hline
\end{tabular}




\begin{tabular}{|c|c|c|c|c|c|}
\hline mo & INST & 4.1 & $4.2 \mathrm{a}$ & $4.2 \mathrm{~b}$ & $4.2 \mathrm{c}$ \\
\hline 1 & ABBOTI VPS & EVERY RUN & AGENT CALIBRATORS & YES & $1 / 2$ \\
\hline 2 & PARALLEL & DAILY & SERUH BASED & VES & $1 / 2$ \\
\hline 3 & PERSPECTIVE & DAILY & SERUM BASED & YES & $1 / 2$ \\
\hline 4 & EMCORE II & EVERY RUN / 30 TESTS & SERUM & YES & $1 / 16$ \\
\hline 5 & SPIRIT & UNDER INVESTIGATION & EITHER & YES & $1 / 3$ \\
\hline 6 & HITACHI 704 & DAILY REAGENT BLANK & EITHER & YES & $1 / 5$ \\
\hline 7 & HITACHI 705 & DAILY REAGENT BLAMK & EITHER & YES & $1 / 5$ \\
\hline 8 & HITACHI 737 & DAILY REAGENT BLAMK & EITHER & YES & $1 / 5$ \\
\hline 9 & EASY & MOST MONTHLY & SERUM & NO & $2 / 2$ \\
\hline 10 & EPOS & ЕАСН ВАТСH & EITHER & YES & $1 / 6$ \\
\hline 11 & ERIS & MOBT MEEKLYY & EITHER & YES & $1 / 10$ \\
\hline 12 & EXSEL & AS FOR ERIS/EPOS & EITHER & YES & $1 / 16$ \\
\hline 13 & EXTRA & MOST MEEKLY & EITHER & YES & $1 / 20$ \\
\hline 14 & Aus021 & MOST MEEKLYY & EITHER & YES & $1 / 11$ \\
\hline 15 & AUs121 & MOST MEEKLY & EITHER & YES & $1 / 11$ \\
\hline 16 & Aus031 & MOST MEEKLYY & EITHER & YES & $1 / 11$ \\
\hline 17 & AUs131 & MOST MEEKLYY & EITHER & YES & $1 / 11$ \\
\hline 18 & Auso4I & MOST MEEKLYY & EITHER & YES & $1 / 11$ \\
\hline 19 & AUS141 & MOST MEEKLY & EITHER & YES & $1 / 11$ \\
\hline 20 & A45061 & MOST MEEKLYY & EITHER & YES & $1 / 11$ \\
\hline 21 & AU5081 & MOST MEEKLY & EITHER & YES & $1 / 11$ \\
\hline 22 & ASt & 8 HOURS & EITHER & mo & 2 \\
\hline 23 & A58 & 8 HOURS & EITHER & no & 2 \\
\hline 24 & AS ENZYME & 8 HOURS & EITHER & No & 2 \\
\hline 25 & AS LIPID & 8 HOURS & EITHER & No & 2 \\
\hline 26 & MIMI IDEAL & 8 HOURS & EITHER & NO & 2 \\
\hline 27 & AS IDEAL. & 8 HOURS & EITHER & No & 2 \\
\hline 28 & $c \times 3$ & 8 HOURS & EITHER & YES & 2 \\
\hline 29 & $\mathrm{CX} 4$ & UP TO 2 MEEKS & EITHER & YES & $1 / 14$ \\
\hline 30 & Cx5 & UP TO 2 MEEKS & EITHER & YES & $1 / 16$ \\
\hline 31 & $\mathrm{CX7}$ & UP TO 2 WEEXS & EITHER & YES & $1 / 16$ \\
\hline 32 & ASCA & REAGENT DEPENDANT & SERUN & YES & DEP ON CALIBRATOR \\
\hline 33 & CHENILYSER & DAILY & SERUH & YES & $1 / 8$ \\
\hline 34 & SYSTEM & ЕАСН ВАТСH & SERUH & YES & $1 / 8$ \\
\hline 35 & PRISMA II & VARIES & MONE RECOMMENDED & YES & $1 / 2$ \\
\hline 36 & CORNING 580 & ONCE PER DAY & SERUH & YES & $1 / 2$ \\
\hline 37 & Copinine 570 & ONCE/DAY & SERUM & YES & $M / D A$ \\
\hline 38 & CORWING EXPRESS & ONCE/DAY & SERUH & YES & $N / D A$ \\
\hline 39 & CPA & MEEKLY & SERUM & VES & $0 / 6$ \\
\hline 40 & $D A C O S 2$ & 2 MEEKLY DEP ON CHEN & SERUH & YES & $1 / 6$ \\
\hline 41 & DIMENSIOM 380 & 3 MOWTHS & EITHER & YES & $3 / 5$ \\
\hline 12 & DIMENSION 760 & 3 MONTHS & EITHER & YES & $3 / 5$ \\
\hline 43 & BREIWER 6-400 & 2-3 MONTHS & SERUM & YES & $1 / 6$ \\
\hline 14 & GREIMER G-450 & 2-3 MONTHS & SERUM & YES & $1 / 6$ \\
\hline 45 & TANDEN 2 & $2-3$ MONTHS & SERUH & YES & $1 / 6$ \\
\hline 46 & TANDEN 3 & 2-3 MONTHS & SERUH & YES & $1 / 6$ \\
\hline 47 & GEMESIS 21 & 24 HOURS & EITHER & YES & $1 / 5$ \\
\hline 40 & помавсH & 8 HOURS-7 DAYS & EITHER & YES & $1 / 6$ \\
\hline 49 & MONAACH PLUS 2 & 8 MOURS-7 DAYS & EITHER & YES & $1 / 6$ \\
\hline 30 & EKTACHEM DT & 90 DAYS & SERUH & KDDAK CALIBRATORS & $2 / 4$ \\
\hline $5 !$ & EXTACHEM 700 & 90 DAYS & SERUH & KODAK CALIBRATORS & $2 / 4$ \\
\hline 52 & PROBRESS & VARIES & EITHER & YES & $1 / 6$ \\
\hline 53 & SPECIFIC & VARIES & EITHER & YES & $1 / 6$ \\
\hline 54 & MOVA BIOMED $1+1$ & AUTOCAL & MONA CAL IBRANTS & NO & ALL INCLUDED IN PACK \\
\hline 55 & MOVA BIOMED $4+4$ & AUTOCAL & NOVA CALIBRANTS & No & ALL IMCLUDED IN PACK \\
\hline 56 & NOVA BIOMED $5+5$ & AUTOCAL & NOVA CALIBRANTS & No & ALL INCLUDED IN PACK \\
\hline 57 & COBAS FARA & VARIABLE & EITHER & YES & $0 / 8$ \\
\hline 58 & COBAS MIRA & VARIABLE & EITHER & YES & $0 / 8$ \\
\hline 59 & ASSIBT & DAILY & SERUM & YES & 1 \\
\hline 60 & CHEN 1 & 1-4 MEEKS & EITHER & YES & 1 \\
\hline 61 & $R A-1000$ & 2 MEEKS & EITHER & YES & $1 / 6$ \\
\hline 62 & $R A-2 X$ & 2 WEEKS & EITHER & YES & $1 / 6$ \\
\hline 63 & $R A-500$ & 2 MEEKS & SERUM & YES & 116 \\
\hline 64 & $R A-X T$ & 2 WEEKS & EITHER & YES & $1 / 6$ \\
\hline 63 & SMAC II & 96 SAAPLLS & SERUM & YES & 2 \\
\hline 66 & SRA 2000 & SEE SMAC II/RA-1000 & SERUH & YES & 2 \\
\hline 67 & GEN PROFILER & MITH MEM REABENTS & EITHER & & $1 / ? ?$ \\
\hline 68 & ULTROLAB I & W/DA & N/DA & M/DA & N/DA \\
\hline 69 & VITALAB 200 & VARIES & ARuEOUS & YES & $1 / 1$ \\
\hline
\end{tabular}




\begin{tabular}{|c|c|c|c|c|c|c|c|c|}
\hline Mo & IMST & 5.1 & 5.2 & 5.3 & 5.4 & 6.1 & $6.2 \mathrm{a}$ & $6.2 \mathrm{~b}$ \\
\hline 1 & ABBOTT UPS & YES & YES & YES & & BY REPLCUP & NO U/E & NO U/E \\
\hline 2 & PARALLELL & YES & YES & YES & 85800 & YES & 40 MINS & 22 HIMS \\
\hline 3 & PERSPECTIVE & YES & YES & YES & BS 800 & YES & 24 MINS & 12 MINS \\
\hline 4 & ENCORE II & YES & YES & YES & BS 800 & YES & NO U/E & NO U/E \\
\hline s & SPIRIT & ME BELIEVE & YES & WE BELIEVE & GAKMOUN & YES & 25 Nins & 15 nIMS \\
\hline 6 & HITACHI 704 & YES & YES & YES & BS 800 & YES & 25 HIMS & 12 nINS \\
\hline 7 & HITACHI 705 & YES & YES & YES & BS 800 & YES & 25 MIMS & 13 HINS \\
\hline 8 & HITACHI 737 & YES & YES & YES & BS 800 & YES & 30 nINS & 12 nINS \\
\hline 9 & EASY & YES & YES & YES & ? BS 4743 & YES & ALEAYS ON & 8 MINS \\
\hline 10 & EPOS & YES & YES & YES & ? BS 4743 & NO U/E & NO U/E & NO U/E \\
\hline 11 & ERIS & YES & YES & YES & ? BS 4743 & YES & 15 MINS & 11 nINS \\
\hline 12 & EXSEL & YES & YES & YES & ? BS 4743 & YES & 15 MINS & 11 hINS \\
\hline 13 & EXTRA & YES & YES & YES & 7854743 & YES & 15 MINS & 11 NINS \\
\hline 14 & AU5021 & YES & YES & YES & ? BS 4743 & YES & IHR POW OF & 18 HINS \\
\hline 15 & Aus121 & YES & YES & YES & ? BS 4743 & YES & IHR POW OF & 18 MIMS \\
\hline 16 & AU5031 & YES & YES & YES & ? BS 4743 & YES & IHR POW OF & 18 NIMS \\
\hline 17 & AUs131 & YES & YES & YES & ? BS 4743 & YES & IHR POW OF & 18 NINS \\
\hline 18 & Aus041 & YES & YES & YES & ? BS 4743 & YES & IHR POH OF & 18 MINS \\
\hline 19 & Aus141 & YES & YES & YES & ? BS 4743 & YES & IHR POW OF & 18 MINS \\
\hline 20 & AUS061 & YES & YES & YES & ? BS 4743 & YES & IHR POH OF & 18 nINS \\
\hline 21 & Aus001 & YES & YES & YES & $?$ BS 4743 & YES & IHR POW OF & 18 MIMS \\
\hline 22 & AS4 & YES & YES & YES & & YES & ALUAYS ON & 1 HIN \\
\hline 23 & ASB & YES & YES & YES & & YES & ALMAYS ON & 1 MIN \\
\hline 24 & AS ENZYME & YES & YES & YES & & YES & ALNAYS ON & 1 HIN \\
\hline 25 & AS LIPID & YES & YES & YES & & YES & ALMAYS ON & I MIM \\
\hline 26 & MINI IDEAL & YES & YES & YES & & YES & ALIAYYS ON & $1 \mathrm{HIN}$ \\
\hline 27 & AS IDEAL & YES & YES & YES & & YES & ALLAYS ON & 1 MIN \\
\hline 28 & Cx3 & YES & YES & YES & & YES & ALMAYS ON & 《1 MIN \\
\hline 29 & $c \times 4$ & YES & YES & YES & & YES & ALINAYS ON & NO U/E \\
\hline 30 & Cx5 & YES & YES & YES & & YES & ALLAYS ON & <13 HINS \\
\hline 31 & $\mathrm{CX7}$ & YES & YES & YES & & YES & ALLAYS OH & 《I HIN \\
\hline 32 & ASCA & YES & YES & NOT AVAIL & BS 5724 & YES & NO U/E & NO U/E \\
\hline 33 & CHEWILYSER & NOT QUOTED & YES & YES & & no & $60 \mathrm{NIN}$ & $30 \mathrm{NIN}$ \\
\hline 34 & SYSTENA & NOT QUOTED & YES & YES & & BY REPLCUP & 75 NINS & $10-20$ NINS \\
\hline 35 & PRISHA II & YES & YES & YES & & YES & 25 MINS & 15 MINS \\
\hline 36 & CORNIMG 580 & YES & YES & YES & TESTED JSI & YES & 60 NINS & 15 MINS \\
\hline 37 & ConwING 570 & YES & YES & YES & TESTED JSI & YES & 15 MINS & 5 KINS \\
\hline 38 & CORNING EXPRESS & YES & YES & YES & IEC 601 & YES & NO U/E & NO U/E \\
\hline 39 & CPA & YES & YES & YES & & YES & NO U/E & NO U/E \\
\hline 40 & DACOS 2 & YES & YES & YES & & YES & 32 NINS & 12 nINS \\
\hline 41 & DIMENSIOW 380 & YES & UN DEVELOP & YES & & YES & 8 MINS & 7 Hins \\
\hline 42 & DIMEWSIOW 760 & YES & UN DEVELOP & YES & & YES & 8 HINS & 7 HINS \\
\hline 43 & SREIMER 6-400 & YES & YES & YES & ? BS 800 & YES & 15 MINS & 15 MINS \\
\hline 44 & GREIMER 6-450 & YES & YES & YES & ? 85800 & YES & 12 hINS & 12 HINS \\
\hline 45 & TAMDEH 2 & YES & YES & YES & ? BS 800 & YES & 12 Mins & 12 HINS \\
\hline 46 & TAMDEN 3 & YES & YES & YES & ? BS 800 & YES & 12 MINS & 12 MIMS \\
\hline 47 & BENESIS 21 & YES & YES & YES & BS 5724 & YES & 13 MINS & 13 MINS \\
\hline 48 & MONARCH & YES & YES & YES & BS 5724 & YES & 6 NINS & 6 MINS \\
\hline 49 & MONARCH PLUS 2 & YES & YES & YES & BS 5724 & YES & 6 HINS & 6 MINS \\
\hline 50 & EXTACHEN DT & YES & YES & YES & & YES & ALLAYS ON & 9 nINs \\
\hline 51 & EXTACHEM 700 & YES & YES & YES & & YES & ALWAYS ON & 10 MINS \\
\hline 52 & PROGRESS & YES & YES & YES & & YES & ALUAYS ON & 9 nIns \\
\hline 53 & SPECIFIC & YES & YES & YES & & YES & ALLAYS ON & 9 NINS \\
\hline 54 & NOVA BIOMED $1 ; 1$ & YES & YES & YES & & YES & NO U/E & NO U/E \\
\hline 55 & MOVA BIOMED $4+1$ & YES & YES & YES & & YES & NO U/E & NO U/E \\
\hline 56 & MOVA BIOMED S+5 & YES & YES & YES & & YES & NO U/E & NO U/E \\
\hline 57 & COBAS FARA & YES & YES & YES & BS 5724 & YES & 12 HINS & 7 HINS \\
\hline 58 & COBAS MIRA & YES & YES & YES & BS 5724 & YES & 12 MIMs & 7 HIMS \\
\hline 59 & A5SIST & YES & YES & YES & & YES & NO U/E & NO U/E \\
\hline 60 & CHEN 1 & YES & YES & YES & & YES & 30 MINS & 15 niws \\
\hline 61 & $R A-1000$ & YES & YES & YES & & YES & ALHAYS ON & 3.5 NIMS \\
\hline 62 & $R A-2 X$ & YES & YES & YES & & YES & ALNAYS ON & 3.5 MINS \\
\hline 63 & $R A-500$ & YES & YES & YES & & YES & NO U/E & WO U/E \\
\hline 64 & $R A-X T$ & YES & YES & YES & & YES & ALMAYS ON & $3.5 \mathrm{nIN}$ \\
\hline 65 & SHAC II & YES & YES & YES & & YES & 35 MINS & 20 MINS \\
\hline 66 & SeA 2000 & YES & YES & YES & & YES & ALIAAYS ON & S+ HINS \\
\hline 67 & BEM PROFILER & YES & Mo & YES & & YES & WO U/E & NO U/E \\
\hline 68 & ULTROLAB I & N/DA & YES & YES & N/DA & YES & W/DA & N/DA \\
\hline 69 & VITALAB 200 & YES & YES & ? YES & $85800 \quad 4743$ & YES & MO U/E & WO U/E \\
\hline
\end{tabular}




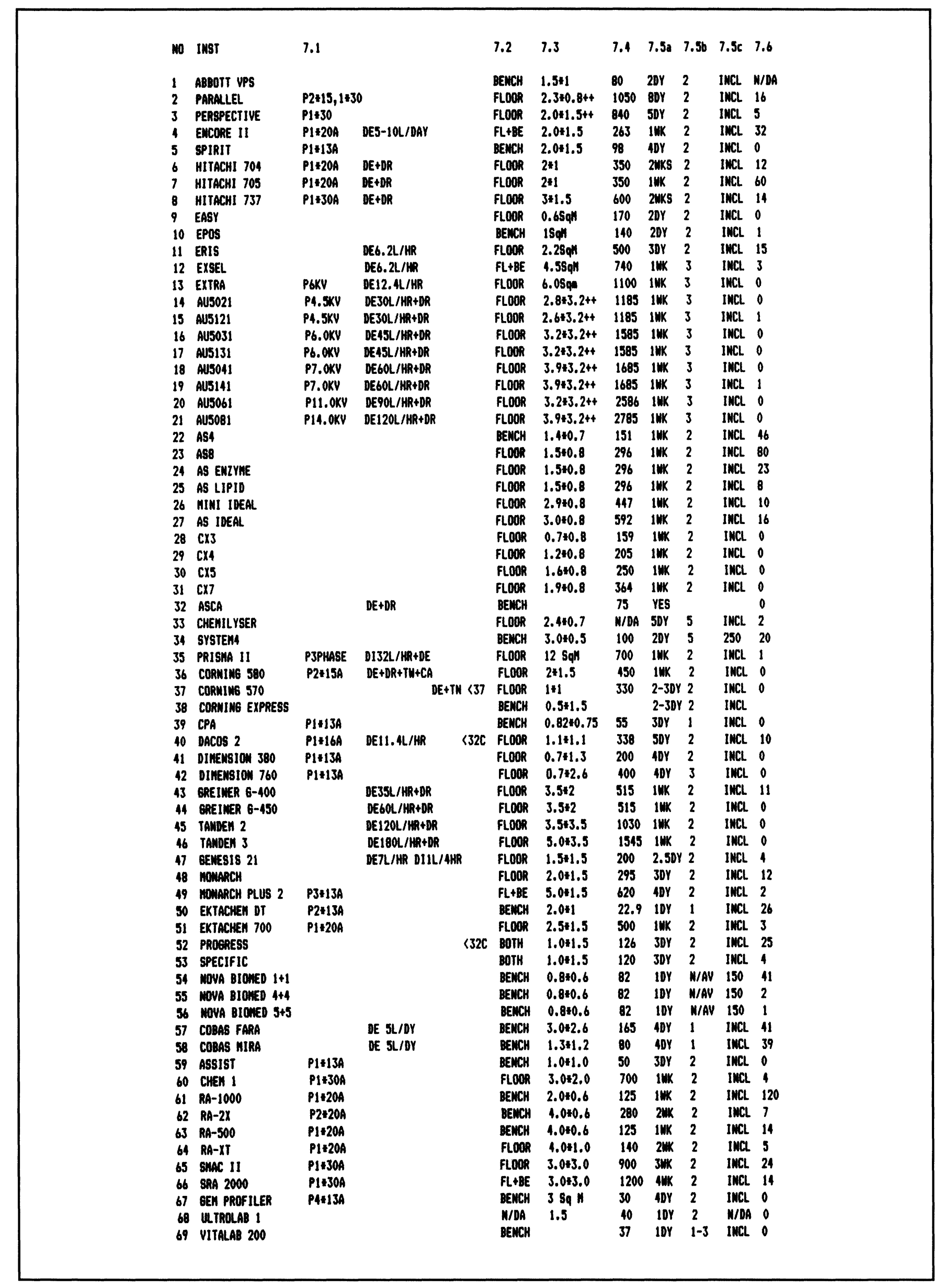




\begin{tabular}{|c|c|c|c|c|c|c|c|c|c|}
\hline No & IMST & $8.1 \mathrm{a}$ & 8.16 & 8.2 & 8.3 & 8.4 & 8.5 & 8.6 & \\
\hline 1 & ABBOTT UPS & N/DA & H/DA & N/DA & No & GПTH & No & TYR & EN \\
\hline 2 & Parallel & $30 \mathrm{HR} / \mathrm{YR}$ & $1 \mathrm{HR}$ & 1 LEVELS & SPARES & IYR & SPARES & TYR & EN \\
\hline 3 & PERSPECTIVE & $20 \mathrm{HR} / \mathrm{YR}$ & 1 HR & 1 LEVELS & no & IYR & SPARES & TYR & EN \\
\hline 4 & EMCORE II & $440.50 Y / Y R$ & 10 Mins & FULL 4368 PH 1836 & no & IVR & No & I7YR & \\
\hline 5 & SPIRIT & $3 * 0.50 Y / Y R$ & 30 HIMS & FULL 3500 & SPARES & IYR & SPARES & ITYR & \\
\hline 6 & HITACHI 704 & $20 \mathrm{HR} / \mathrm{KR}$ & 20 nIMs & FULL 63603 LEVELS AVAIL 9.6 & ! No & IYR & No & TYR & EN \\
\hline 7 & HITACHI 705 & $20 \mathrm{HR} / \mathrm{YR}$ & 20 HIMS & FULL 64203 LEVELS AVAIL & no & IYR & No & TYR & En \\
\hline 8 & HITACHI 737 & $20 \mathrm{HR} / \mathrm{YR}$ & 30 MINS & FULL 115803 LEVELS AVAIL & 6 No & IYR & No & 7YR & EN \\
\hline 9 & EASY & N/DA & $N / D A$ & & SPARES & IVR & SPARES & ZIOYR & \\
\hline 10 & EPOS & 6 HR/VR & & & No & IVR & SPARES & J1OYR & \\
\hline 11 & ERIS & $13 \mathrm{HR} / \mathrm{YR}$ & & & SPARES & IYR & SPARES & J1OYR & \\
\hline 12 & EXSEL & $20 \mathrm{HR} / \mathrm{YR}$ & & & SPARES & IYR & SPARES & J1OYR & \\
\hline 13 & EXTRA & 26 HR/YR & & & SPARES & IYR & SPARES & JIOKR & \\
\hline 14 & AU5021 & $16 \mathrm{HR} / \mathrm{YR}$ & $M / D A$ & & SPARES & IVR & SPARES & J1OYR & \\
\hline 15 & AU5121 & $16 \mathrm{HR} / \mathrm{YR}$ & & & SPARES & IYR & SPARES & MoYR & \\
\hline 16 & AUs031 & $16 \mathrm{HR} / \mathrm{YR}$ & & & SPARES & IVR & SPARES & DIOYR & \\
\hline 17 & AU5131 & 18 HR/YR & & & SPARES & IYR & SPARES & JIOYR & \\
\hline 18 & AU5041 & $20 \mathrm{HR} / \mathrm{YR}$ & & & SPARES & IVR & SPARES & JIOYR & \\
\hline 19 & Aus141 & $20 \mathrm{HR} / \mathrm{YR}$ & & & SPARES & IYR & SPARES & J1OYR & \\
\hline 20 & AU5061 & $24 \mathrm{HR} / \mathrm{YR}$ & & & SPARES & IYR & SPARES & J1OYR & \\
\hline 21 & AU5081 & 28 HR/YR & & & SPARES & IYR & SPARES & IIOYR & \\
\hline 22 & AS4 & $1 \mathrm{OY} / \mathrm{YrR}$ & 5 HINS & FULL 1652 & SPARES & IYR & SPARES & $7 Y R$ & EN \\
\hline 23 & ASO & 1 DY/YR & 5 MINS & FULL 6496 & SPARES & IYR & SPARES & TYR & EN \\
\hline 24 & AS ENZYME & $1 \mathrm{DY} / \mathrm{YR}$ & 5 MIMS & FULL 8094 & SPARES & IYR & SPARES & TYR & EN \\
\hline 25 & AS LIPID & I DY/YR & 5 MINS & FULL 7295 & SPARES & IYR & SPARES & TYR & EY \\
\hline 26 & HINI IDEAL & 1 DY/YR & 5 MINS & FULL 11947 & SPARES & IYR & SPARES & TYR & EN \\
\hline 27 & AS IDEAL & I DY/YR & 5 HINS & FULL 15389 & SPARES & IYR & SPARES & TYR & EN \\
\hline 28 & $\mathrm{Cx3}$ & I DY/YR & 2 MINS & FULL 4000 & SPARES & IYR & SPARES & TYR & EN \\
\hline 29 & CX4 & $1 \mathrm{DY} / \mathrm{KR}$ & N/DA & N/DA & SPARES & IVR & SPARES & TYR & EN \\
\hline 30 & $C \times 5$ & $1 D Y / Y R$ & N/DA & N/DA & SPARES & IYR & SPARES & $7 Y R$ & EN \\
\hline 31 & $\mathrm{Cx} 7$ & $1 \mathrm{DY} / Y \mathrm{YR}$ & W/DA & $N / D A$ & SPARES & IYR & SPARES & TYR & EM \\
\hline 32 & ASCA & $1 \mathrm{DY} / \mathrm{YR}$ & 5 hINS & & No & IYR & No & 10YR & En \\
\hline 33 & CHENILYSER & $2 D Y / Y R$ & 25 MINS & FULL 4500 & NO & IYR & no & IOYR & \\
\hline 34 & SYSTEM4 & 2 DY/YR & 25 nINS & FULL 2500 & No & IVR & NO & 10YR & \\
\hline 35 & PRISHA II & $40 \mathrm{HR} / \mathrm{YR}$ & 30 MINS & FULL 140005 LEVELS AVAIL & SPARES & IVR & SPARES & 1OYR & \\
\hline 36 & CORNING 580 & $26 \mathrm{HR}$ & 30 HINS & FULL 11200 & SPARES & IYR & SPARES & TYR & EN \\
\hline 37 & CORWING 570 & N/DA & 30 MINS & FULL 7500 & SPARES & IYR & SPARES & TYR & EM \\
\hline 38 & CORWING EXPRESS & $16 \mathrm{HR} / \mathrm{YR}$ & 15 MINS & FULL 3000 & SPARES & IYR & SPARES & TYR & EN \\
\hline 39 & CPA & $12 \mathrm{HR} / \mathrm{YR}$ & 2 MIN & FULL 2600 & No & ISE IY & No & INDEF & \\
\hline 40 & DACOS 2 & $20 \mathrm{HR} / \mathrm{KR}$ & $12 \mathrm{NIN}$ & FULL 80002 LEVELS AVAIL & No & ISE IY & no & INDEF & \\
\hline $4 !$ & DIMENSIOW 380 & 12 HR/YR & 10 MINS & & T SPARES & $1-2 Y R$ & SPARES & IOYR & \\
\hline 42 & DIMENSIOW 760 & 12 HR/YR & 10 MIns & & \% SPARES & $1-2 Y R$ & SPARES & 10YR & \\
\hline 43 & GREINER 6-400 & 2 DY/YR & & FULL 8200 LEVELS AVAIL & No & IYR & No & J10YR & \\
\hline 44 & GREINER O-450 & 2 DY/YR & & FULL 82004 LEVELS AVAIL & No & IYR & no & I1OYR & \\
\hline 45 & TAMDEN 2 & 2 DY/YR & & FULL 12000 \& LEVELS AVAIL & NO & IYR & No & SHOYR & \\
\hline 46 & TAMDEN 3 & 2 DY/YR & & FULL 18000 LEVELS AVAIL & MO & IYR & No & nIOYR & \\
\hline 47 & GEWESIS 21 & $2 D Y / Y R$ & & FULL 4500 PK 550 & SPARES & IYR & SPARES & IOYR & \\
\hline 48 & MOMARCH & $2 D Y / Y R$ & 10 hINS & FULL 5500 PH 540 & SPARES & IYR & SPARES & LOYR & \\
\hline 49 & mOMARCH PLUS 2 & $4 \mathrm{DY} / \mathrm{YR}$ & 20 nINS & FULL $12700 \mathrm{PM} 1080$ & SPARES & IYR & SPARES & IOYR & \\
\hline 50 & EXTACHEN OT & 4 HR/YR & 5 MINS & FULL 680 & SPARES & IVR & SPARES & IOYR & En \\
\hline 51 & EXTACHEM 700 & $1 \mathrm{BY} / \mathrm{YR}$ & 10 MIns & FULL 8500 & SPARES & IYR & SPARES & 10YR & EN \\
\hline 52 & PRDORESS & & & PH 3.5\% FULL 9. & \% no & IYR & No & J5YR & EN \\
\hline 53 & SPECIFIC & & & FULL 9. & no & IYR & мо & >SYR & EN \\
\hline 54 & MOVA BIOWED $1+1$ & & & & \% mo & IYR & YES & I7YR & \\
\hline 55 & MOVA BIOMED A+4 & & & & 4 No & IYR & YES & ITYR & \\
\hline 56 & MOVA BIOHED S+5 & & & & \% No & IYR & YES & ITYR & \\
\hline 57 & COBAS FARA & & & FULL 4200 & Mo & IYR & No & STYR & EN \\
\hline 58 & COBAS MIRA & & & FULL 2800 & No & IYR & no & ITYR & EN \\
\hline 59 & A8SIGT & 0 & 15 MIN & NO DATA & No & IYR & no & TYR & EN \\
\hline 60 & CHEN 1 & $1 \mathrm{OY} / \mathrm{YR}$ & $<5$ HIN & FULL 9950 & SPARES & IYR & SPARES & TYR & EN \\
\hline 61 & $R A-1000$ & 0 & $15 \mathrm{MIM}$ & FULL 3647 & NO & IYR & MO & TYR & EN \\
\hline 62 & $R A-2 X$ & 0 & $2 * 15$ NIN & FULL 8546 & No & IVR & wo & TYR & EN \\
\hline 63 & $R A-500$ & 0 & $15 \mathrm{MIN}$ & FULL 3647 & mo & IYR & Mo & TYR & EN \\
\hline 64 & $R A-X T$ & 0 & 15 MIN & FULL 5968 & no & IVR & wo & TYR & En \\
\hline 65 & $\operatorname{senc}$ II & \& DY/YR & 1 HR & FULL 17244 & SPARES & IVR & SPARES & TYR & EN \\
\hline 66 & SRA 2000 & 4 DY/YR & 1 H & FILL 20063 & SPAPES & IYR & SPARES & TYR & EN \\
\hline 67 & GEM PROFILER & $240.50 \mathrm{r}$ & 0 & BASIC OX FULL 1 & 7 no & IYR & No & INDEFF & \\
\hline 68 & ULTROLAB 1 & & & & q wo & IVR & N/DA & W/DA & \\
\hline 69 & VITALAB 200 & 2 DY/YR & 10 MING & FULL 1500 PH 480 & No & IVR & No & IOYR & \\
\hline
\end{tabular}




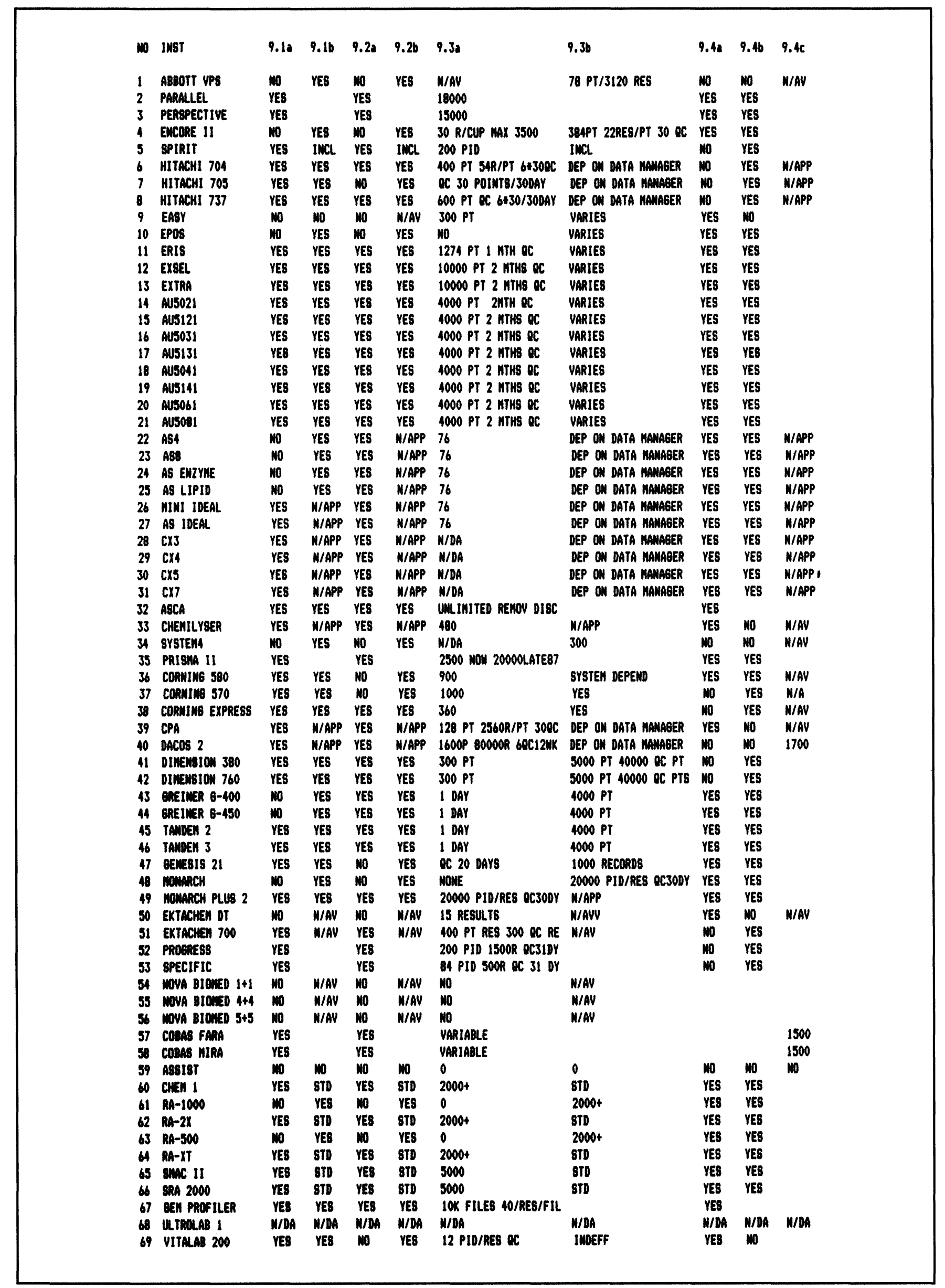




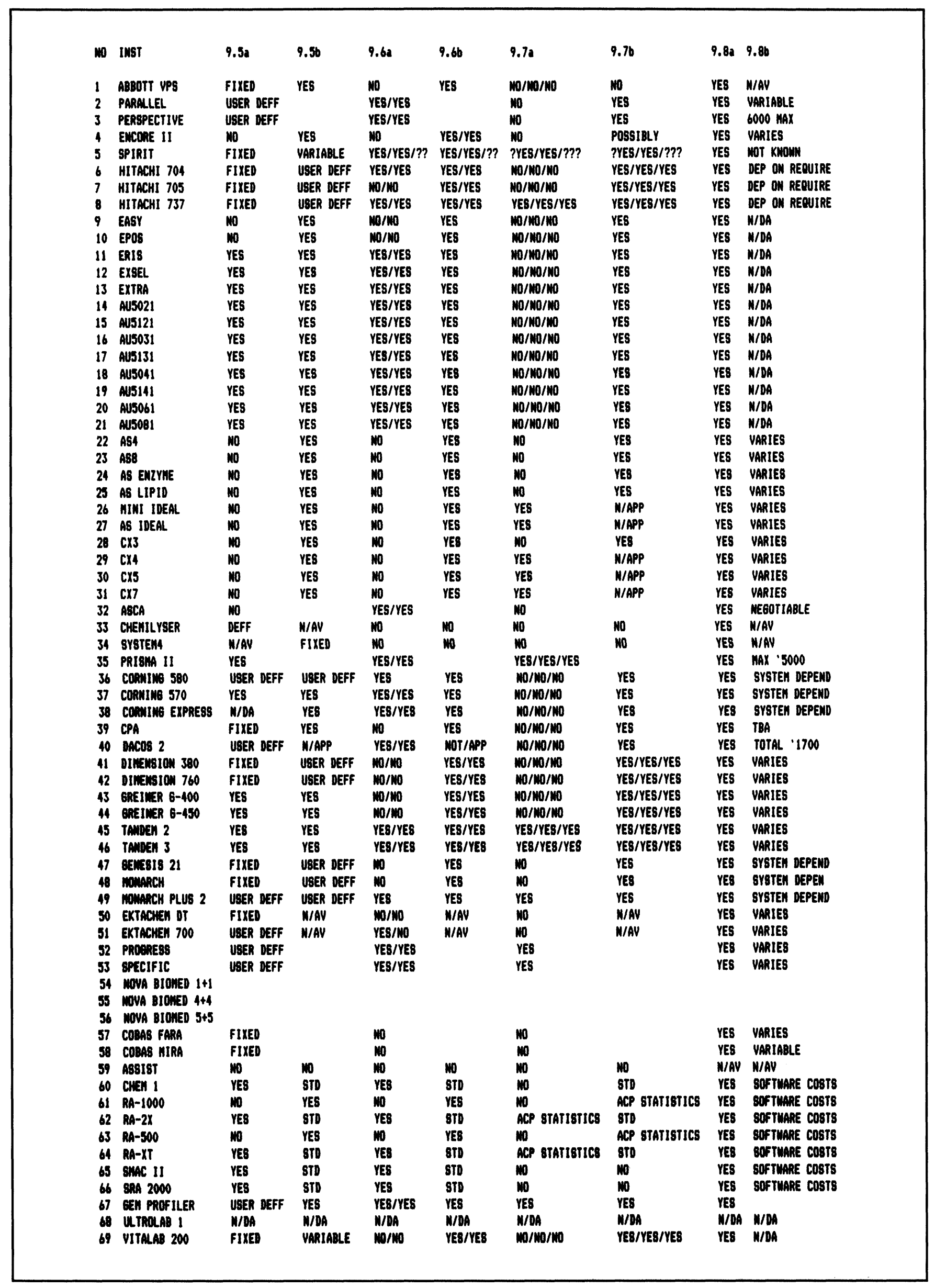




\begin{tabular}{|c|c|c|c|c|c|c|c|c|c|c|c|}
\hline$M$ & INST & 10.1 & $10.2 \mathrm{a}$ & 10.26 & 10.3 U/E & 10.3 LFT & 10.3BONE & 10.3CARD & $10.3 \mathrm{HISC}$ & 10.3 GLU & $10.3 \mathrm{ALL}$ \\
\hline 1 & ALBOTT VPS & 22000 & N/AV & 4000 & MO U/E & N/DA & N/DA & N/DA & N/DA & $N / D A$ & N/DA \\
\hline 2 & PARALLEL & 217500 & InCL & INCL & 11.67 & 13.06 & 3.87 & 11.23 & 12.27 & 2.10 & 54.20 \\
\hline 3 & PERSPECTIVE & 115000 & INCL & INCL & 9.28 & 16.07 & 4.71 & 14.28 & 12.35 & 1.40 & 56.69 \\
\hline 4 & Excone II & 49500 & $N / A$ & $2-5000$ & NOU/E & 18.63 & 10.50 & 14.49 & 12.42 & N/DA & NO U/E \\
\hline 5 & SPIRIT & 35000 & INCL & $\mathrm{INCL}$ & 23.45 & 17.31 & 13.30 & 18.44 & 25.13 & N/DA & 97.63 \\
\hline 6 & HITACHI 704 & 49000 & 10000 & DEPENDS & 21.40 & 25.45 & 14.28 & 13.29 & 31.58 & 2.73 & 96.97 \\
\hline 7 & HITACHI 705 & APP35000 & 12300 & DEPENDS & 25.90 & 24.80 & 13.80 & 13.40 & 30.70 & 5.11 & 96.90 \\
\hline 8 & HITACHI 737 & 129000 & INCL & IMCL & 20.56 & 22.11 & 12.53 & 12.68 & 20.67 & 4.46 & 81.62 \\
\hline 9 & EASY & 37500 & INCL & VARIES & 189.00 & 375.00 & 196.00 & 189.00 & 249.50 & 22.00 & 930.90 \\
\hline 10 & EPOS & 37500 & N/AV & VARIES & WO U/E & 11.5 & 6.20 & 5.70 & 14.33 & 2.00 & NO U/E \\
\hline 11 & ERIS & 80000 & 16500 & VARIES & 10.78 & 15.48 & 10.44 & 7.65 & 17.28 & 3.42 & 56.67 \\
\hline 12 & EXBEL & 150000 & IIMCL & IMCL & 10.78 & 11.50 & 6.20 & 5.70 & 14.33 & 2.00 & 44.72 \\
\hline 13 & EXTEA & 180000 & IXCL & IMCL & 10.78 & 15.48 & 10.44 & 7.65 & 17.28 & 3.42 & 56.60 \\
\hline 14 & AUs021 & 165000 & 10000 & INCL. & 9.04 & 14.08 & 9.00 & 7.20 & 15.20 & 1.60 & 46.72 \\
\hline 15 & MUS121 & 165000 & 10000 & IMCL & 9.04 & 14.08 & 9.00 & 7.20 & 15.20 & 1.60 & 46.72 \\
\hline 16 & AUS031 & 210000 & 10000 & INCL. & 9.04 & 14.08 & 9.00 & 7.20 & 15.20 & 1.60 & 46.72 \\
\hline 17 & AU5I3! & 210000 & IMCL & IMCL & 9.04 & 14.08 & 9.00 & 7.20 & 15.20 & 1.60 & 46.72 \\
\hline 18 & AUSO41 & 250000 & INCL & IMCL & 9.04 & 14.08 & 9.00 & 7.20 & 15.20 & 1.60 & 46.72 \\
\hline 19 & AUs141 & 250000 & INCL & INCL & 9.04 & 14.08 & 9.00 & 7.20 & 15.20 & 1.60 & 46.72 \\
\hline 20 & AUs061 & 320000 & IMCL & INCL & 9.04 & 14.08 & 9.00 & 7.20 & 15.20 & 1.60 & 46.72 \\
\hline 21 & aUs081 & 370000 & IMCL & INCL & 9.04 & 14.08 & 9.00 & 7.20 & 15.20 & 1.60 & 46.72 \\
\hline 22 & A84 & 30000 & IMCL & $3-15000$ & N/AV & N/AV & N/AV & N/AV & N/AV & N/AV & N/AV \\
\hline 23 & AS8 & 49950 & INCL & INCL & N/AV & N/AV & N/AV & N/AV & N/AV & N/AV & N/AV \\
\hline 24 & AS ENZYME & 65950 & InCL & IMCL & W/AV & W/AV & N/AV & N/AV & N/AV & N/AV & N/AV \\
\hline 25 & E LPDD & 59950 & INCL & INCL & N/AV & N/AV & N/AV & N/AV & N/AV & N/AV & N/AV \\
\hline 26 & HINI IOEAL & 99500 & IMCL & IMCL & N/AV & N/AV & N/AV & N/AV & N/AV & W/AV & N/AV \\
\hline 27 & AS IDEA & 120000 & INCL & INCL & N/AN & N/AV & N/AV & N/AV & N/AV & N/AV & N/AV \\
\hline 28 & cxs & 39950 & INCL & $3-15000$ & N/AV & N/AV & N/AV & N/AV & N/AV & N/AV & N/AV \\
\hline 29 & cx4 & APP 67500 & & $3-15000$ & N/AV & N/AV & M/AV & M/AV & N/AV & N/AV & N/AV \\
\hline 30 & cxs & APP72500 & IMCL & $3-15000$ & N/AN & N/AV & N/AV & N/AV & N/AV & N/AV & N/AV \\
\hline 31 & $c \times 7$ & APP95000 & INCL & $3-15000$ & N/AV & N/AV & N/AV & N/AV & N/AV & N/AV & N/AV \\
\hline 32 & ASCA & 16000 & W/A & INCL & N/AV & W/AV & N/AV & N/AV & N/AV & N/AV & N/AV \\
\hline 33 & CHEMILYSER & 60000 & & & 6.00 & $N / D A$ & 4.00 & $N / D A$ & H/DA & $N / D A$ & $N / D A$ \\
\hline 34 & SYSTERA & 35000 & & & N/BA & N/DA & $N / D A$ & N/DA & N/DA & N/DA & $N / D A$ \\
\hline 38 & PRIE⿵⺆ II & 200000 & N/AV VET & IMCL & 7.35 & 24.00 & 8.60 & 17.65 & 16.00 & $N / D A$ & 73.60 \\
\hline 36 & Conewing 590 & 140000 & INCL & IMCL & 24.00 & 28.00 & 16.00 & 25.00 & 12.00 & N/DA & 84.00 \\
\hline 37 & ConuING 570 & 75000 & INCL & $A 915000$ & $N / D A$ & N/DA & N/DA & N/DA & N/DA & N/DA & N/DA \\
\hline 38 & CORNINS EXPRESS & 30000 & $N / A$ & SYS DEP & NO U/E & N/OA & $N / D A$ & N/DA & N/OA & $N / D A$ & N/DA \\
\hline 39 & CPA & 30000 & N/AV & VARIABLE & WUVE & 11.49 & 11.88 & 10.79 & 15.50 & 1.49 & NO U/E \\
\hline 40 & $\operatorname{Daccs} 2$ & 85000 & 14800 & VARIABLE & 16.33 & 19.10 & 12.14 & 12.46 & 15.75 & 3.01 & 78.79 \\
\hline 41 & Binsusion 380 & 50000 & IMCL & 5000 & 14.91 & 80.00 & 30.00 & 40.00 & 40.00 & 5.00 & 184.91 \\
\hline 42 & Dinesusion 760 & 90000 & INCL & INCL & 14.91 & 80.00 & 30.00 & 40.00 & 40.00 & 5.00 & 184.91 \\
\hline 43 & BenEITER 6-400 & 60000 & 9000 & 14000 & 12.90 & 17.36 & 4.59 & 9.98 & 22.77 & 0.95 & 62.81 \\
\hline 44 & EREIIIRR 6-450 & 68000 & 9000 & 14000 & 12.90 & 17.36 & 4.59 & 9.98 & 22.77 & 0.95 & 62.81 \\
\hline 40 & TAUE 2 & 130000 & 9000 & IMCL & 12.90 & 17.36 & 4.59 & 9.98 & 22.77 & 0.95 & 62.81 \\
\hline 16 & Tamoen 3 & 180000 & 9000 & IMCL & 12.90 & 17.36 & 4.59 & 9.98 & 22.77 & 0.95 & 62.81 \\
\hline 4 & Eassis 21 & 47000 & 3000 & 5500 & 19.50 & 17.80 & 11.50 & 24.50 & 19.20 & 0.80 & 93.30 \\
\hline 48 & моманси & 60000 & 5000 & 5500 & 9.60 & 11.60 & 5.50 & 24.50 & 19.50 & 0.80 & 63.50 \\
\hline 49 & mounch PLUS 2 & 136000 & 10000 & IIMCL & 9.60 & 11.60 & 5.50 & 24.50 & 11.30 & 0.80 & 63.50 \\
\hline 50 & EXTACHEN or & 9100 & IMCL & W/AP & 216.60 & 343.00 & N/AW & 195.00 & 195.00 & $N / D A$ & 985.60 \\
\hline 51 & Exтасиен 700 & 112000 & IMCL & M/APP & 84.00 & 134.00 & 64.00 & 70.00 & 70.00 & 14.00 & 436.00 \\
\hline 52 & PRoeritss & 49500 & 5000 & VARIES & 7.0 & 5.32 & 2.96 & 2.10 & 7.82 & 0.77 & 23.00 \\
\hline 53 & SPECIFIC & 31500 & 5000 & VARIES & 7.0 & 5.32 & 2.96 & 2.10 & 7.82 & 0.77 & 23.00 \\
\hline 54 & NOVA BIOHED $1+1$ & 9545 & VARious & N/DA & N/AV & $*$ & + & + & $*$ & + & $*$ \\
\hline 56 & WOVA BIOWED $4+4$ & 17990 & VaRious & N/DA & N/AV & 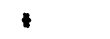 & 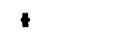 & 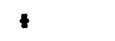 & + & + & $\star$ \\
\hline 56 & WOVA BIONEO $5+5$ & 14745 & VARIous & N/DA & N/AV & $\star$ & $*$ & $*$ & 4 & $\star$ & $*$ \\
\hline 57 & COBAS FARA & 51100 & 8500 & VARIES & 15.60 & 7.40 & 6.90 & 6.40 & 14.30 & N/DA & 50.60 \\
\hline 58 & COBAS MIRA & 30700 & 8500 & VARIES & 15.60 & 9.40 & 6.90 & 7.40 & 14.30 & N/DA & 53.60 \\
\hline 59 & Ass18T & 16000 & N/AV & N/AV & N/DA & $N / D A$ & N/DA & N/DA & $N / D A$ & N/DA & N/DA \\
\hline 60 & CHEN 1 & 160000 & INCL & InCL & 3.50 & 21.00 & 9.00 & 12.00 & 16.00 & N/DA & 61.50 \\
\hline 61 & $R A-1000$ & 40000 & 5000 & 6000 & 8.83 & 25.87 & 9.35 & 23.59 & 17.27 & $N / D A$ & 84.91 \\
\hline 62 & $R A-2 X$ & 90000 & Imel & Imal & 8.83 & 25.87 & 9.35 & 23.59 & 17.27 & N/DA & 84.91 \\
\hline 63 & $R A-500$ & 25000 & N/AV & 10000 & WO U/E & 25.87 & 9.35 & 23.59 & 17.27 & N/DA & WO U/E \\
\hline 64 & $R A-X T$ & 59000 & 8000 & Ine & 8.83 & 23.87 & 9.35 & 23.59 & 17.27 & $N / D A$ & 84.91 \\
\hline 65 & $\operatorname{sinc}$ II & 160000 & IMCL & IMCL & 30.10 & 30.10 & 30.10 & 30.10 & 30.10 & $N / B A$ & 30.10 \\
\hline 66 & $\sin 2000$ & 225000 & INCL & InCL & 30.10 & 30.10 & 30.10 & 30.10 & 30.10 & N/DA & 30.10 \\
\hline 67 & EEN PROFILER & 39950 & M/AV & IMCL & WU U/E & N/AV & N/AN & N/AV & M/AV & N/AV & N/AV \\
\hline $\begin{array}{l}68 \\
6\end{array}$ & $\begin{array}{l}\text { UTACLAB } 1 \\
\text { UITALAB } 200\end{array}$ & $\begin{array}{l}\text { N/DA } \\
13773\end{array}$ & $\begin{array}{l}\text { W/DA } \\
3900\end{array}$ & $\begin{array}{l}\text { W/DA } \\
\text { W/OA }\end{array}$ & $\begin{array}{l}\text { W U/E } \\
W / B A\end{array}$ & $\begin{array}{l}16.00 \\
\text { N/BA }\end{array}$ & $\begin{array}{l}5.00 \\
N / D A\end{array}$ & $\begin{array}{l}9.00 \\
N / D A\end{array}$ & $\begin{array}{l}16.0 \\
N / D A\end{array}$ & $\begin{array}{l}\text { N/DA } \\
\text { N/BA }\end{array}$ & $\begin{array}{l}\text { N/DA } \\
\text { W/DA }\end{array}$ \\
\hline
\end{tabular}




\begin{tabular}{|c|c|c|c|c|c|c|c|c|c|c|}
\hline mo & INBT & 10.4 U/E & 10.4 LFT & $10.480 \mathrm{mE}$ & 10.4CARD & 10.4nI8C & $10.1 \mathrm{ALL}$ & $10.5 \mathrm{~s}$ & 10.56 & 10.56 \\
\hline 1 & ABB:OTT UPS & N/DA & N/DA & M/DA & $M / D A$ & M/OA & M/DA & $M / D A$ & W/DA & $M / D A$ \\
\hline 2 & PARRLLLL & 0 & 0 & 0 & 0 & 0 & 0 & $1.8 \mathrm{~m}$ & $42 p$ & IOML. 24HR \\
\hline 3 & PERSPECTIVE & 3.50 & 4.90 & 2.80 & 2.10 & 2.10 & 72.09 & $1.2 \mathrm{~m}$ & $42 p$ & 10Min 24tin \\
\hline 1 & ENCOAE II & WO U/E & 0.93 & 0.93 & 0.93 & 0.93 & NO U/E & $0.25 \mathrm{ml}$ & 91P & IOWL JODAYS \\
\hline 5 & SPIRIT & 2.15 & 2.15 & 2.15 & 2.15 & 2.15 & 2.15 & $1.1 \mathrm{~mL}$ & 90P & 10mL10DY/SmL10Y \\
\hline 6 & HITACHI 704 & 1.25 & 1.25 & 1.25 & 1.25 & 1.25 & 1.25 & $0.30 \mathrm{ML}$ & $98 P$ & 3XiL 2DAYS \\
\hline 7 & HITACHI 705 & 1.25 & 1.25 & 1.25 & 1.25 & 1.25 & 1.25 & $0.44 \mathrm{~m}$ & $98 \mathrm{P}$ & 3YiL 2DAYS \\
\hline 8 & HITACHI 737 & 1.25 & 1.25 & 1.25 & 1.25 & 1.25 & 1.25 & $0.22 \mathrm{NL}$ & $98 P$ & 3hi. 2DAYS \\
\hline 9 & EASY & 0 & 0 & 0 & 0 & 0 & 0 & InL & $80 P$ & IIIL. INK \\
\hline 10 & EPOS & NO U/E & 0 & 0 & 0 & 0 & 0 & $0.2 \mathrm{~mL}$ & $45 P$ & SIII IVK \\
\hline 11 & ERIS & 10.00 & 17.50 & 10.00 & 7.50 & 7.50 & 42.50 & $0.2 m$ & 45P & sim. INK \\
\hline 12 & EXSEL & 10.00 & 0 & 0 & 0 & 0 & 10.00 & $0.3 \mathrm{ML}$ & $45 P$ & 5WI INK \\
\hline 13 & EXTRA & 10.00 & 17.50 & 10.00 & 7.50 & 7.50 & 42.50 & $0.36 \mathrm{ML}$ & 45P & 5in. INK \\
\hline 14 & AUS021 & 0 & 0 & 0 & 0 & 0 & 0 & 2iil & $45 P$ & 5ML JWK \\
\hline 15 & AUs121 & 0 & 0 & 0 & 0 & 0 & 0 & $2 \mathrm{~mL}$ & 45P & 5in. IVK \\
\hline 16 & AUs031 & 0 & 0 & 0 & 0 & 0 & 0 & $2 \pi i k$ & $45 P$ & 5in. IWK \\
\hline 17 & AUs131 & 0 & 0 & 0 & 0 & 0 & 0 & $2 m$ & 45P & 5im. IIIK \\
\hline 18 & AUs041 & 0 & 0 & 0 & 0 & 0 & 0 & $2 m$ & $45 p$ & 5im. IUK \\
\hline 19 & AUs141 & 0 & 0 & 0 & 0 & 0 & 0 & $2 m$ & 45P & 5im. INK \\
\hline 20 & Ausobl & 0 & 0 & 0 & 0 & 0 & 0 & $2 M L$ & $45 P$ & 5ill. IUKK \\
\hline 21 & AUs081 & 0 & 0 & 0 & 0 & 0 & 0 & $2 n i$ & $45 P$ & 5in 1WK \\
\hline 22 & AB4 & 0 & 0 & 0 & 0 & 0 & 0 & W/AV & N/AV & N/AV \\
\hline 23 & ASB & 0 & 0 & 0 & 0 & 0 & 0 & W/AV & M/AV & N/AV \\
\hline 24 & AS ENZYME & 0 & 0 & 0 & 0 & 0 & 0 & N/AV & N/AV & M/AV \\
\hline 25 & AS LIPID & 0 & 0 & 0 & 0 & 0 & 0 & M/AV & M/AV & N/AV \\
\hline 26 & MIWI IDEAL & 0 & 0 & 0 & 0 & 0 & 0 & M/AV & N/AV & W/AV \\
\hline 27 & AS IDEML & 0 & 0 & 0 & 0 & 0 & 0 & M/AV & W/AV & W/AV \\
\hline 28 & $\mathrm{c} \times 3$ & 0 & 0 & 0 & 0 & 0 & 0 & M/AV & N/AV & M/AV \\
\hline 29 & $C \times 4$ & 0 & 0 & 0 & 0 & 0 & 0 & M/AV & N/AV & N/AN \\
\hline 30 & CX5 & 0 & 0 & 0 & 0 & 0 & 0 & M/AV & N/AV & M/AV \\
\hline 31 & $C \times 7$ & 0 & 0 & 0 & 0 & 0 & 0 & N/AV & N/AV & N/AV \\
\hline 32 & ABCA & 2.00 & 2.00 & 2.00 & 2.00 & 2.00 & 2.00 & VARIES & VARIES & \\
\hline 33 & CHENILYSER & W/DA & N/DA & N/DA & N/DA & N/DA & N/DA & N/DA & W/DA & \\
\hline 3 & SYSTEM & N/DA & N/DA & N/DA & $M / D A$ & N/DA & $M / D A$ & N/DA & N/DA & N/DA \\
\hline 35 & PRISTA II & 1.50 & 1.50 & 1.50 & 1.50 & 1.30 & 1.50 & 2mill & DEP & DEPENDS ON CAL \\
\hline 36 & coomings 580 & 0.50 & 0.50 & 0.50 & 0.50 & 0.50 & 0.50 & Sill & $100 p$ & 10ML 2DAYS \\
\hline 37 & CopenIIN 570 & M/DA & M/DA & M/DA & N/DA & $M / D A$ & $N / D A$ & 5im. & $100 p$ & 10MK 2DAYS \\
\hline 39 & COBAING EXPRESS & WO U/E & 2.00 & 2.00 & 2.00 & 2.00 & 2.00 & IMI & $100 p$ & IML. 2DAYS \\
\hline 39 & & WO U/E & M/DA & W/DA & $N / D A$ & N/DA & $N / D A$ & $0.12 \pi \mathrm{L}$ & $22 p$ & Shl 24th \\
\hline 40 & $\operatorname{aAcos} 2$ & N/DA & N/DA & N/DA & M/DA & $M / D A$ & M/DA & $0.82 \% 1$ & $22 \mathrm{p}$ & 3HL 24HR \\
\hline 41 & DINEWBIOW 380 & 6.40 & 6.40 & 6.40 & 6.40 & 6.40 & 6.40 & 0.74 m. & AP270P & 3*1ML BHRS \\
\hline 42 & DITerwsion 760 & 6.40 & 6.40 & 6.40 & 6.40 & 6.40 & 6.40 & $0.74 \mathrm{~m}$ & AP270P & 3*1M 8HAS \\
\hline 43 & BREIIEAR 6-400 & 1.96 & 1.96 & 1.52 & 0.86 & 0.86 & 5.92 & (1) m & VARIES & VARIES \\
\hline 44 & GREINER 6-450 & 1.96 & 1.96 & 1.52 & 0.86 & 0.86 & 5.92 & $\| m$ & VARIES & VARIES \\
\hline 45 & TANDEN 2 & 1.96 & 1.96 & 1.52 & 0.86 & 0.86 & 5.92 & $<2 \mathrm{~m}$ & VARIES & VARIESS \\
\hline 46 & TAMDEN 3 & 1.96 & 1.96 & 1.52 & 0.86 & 0.86 & 5.92 & $<2 m$ & VARIES & VARIES \\
\hline 47 & GEUES18 21 & 0 & 0 & 0 & 0 & 0 & 0 & $0.70 \mathrm{~mL}$ & $20 p$ & SII TDAYS \\
\hline 48 & mominch & 8.70 & 20.30 & 11.60 & 8.70 & 8.70 & 58.00 & $0.30 \mathrm{~mL}$ & 200 & SML TDAYS \\
\hline 49 & MOMACCH PLUS 2 & 8.70 & 20.30 & 11.60 & 8.70 & 8.70 & 59.00 & $0.30 \mathrm{~mL}$ & $20 P$ & Sall TDAYS \\
\hline 50 & EKTACHEY DT & 0 & 0 & 0 & 0 & 0 & 0 & $1.3 \mathrm{~m}$ & IIIP & 3MIL IDAY \\
\hline 51 & EXTACHEN 700 & 0 & 0 & 0 & 0 & 0 & 0 & $0.95 \mathrm{~m}$ & $125 p$ & 3HL IDAY \\
\hline 52 & PRDORESS & 3.00 & 14.00 & 8.00 & 6.00 & 6.00 & 35.00 & W/DA & N/DA & N/DA \\
\hline 53 & SPECIFIC & 3.00 & 14.00 & 8.00 & 6.00 & 6.00 & 35.00 & M/DA & M/DA & $M / D A$ \\
\hline 54 & MOVA BIOWED $1+1$ & N/AV & \& & $\cdot$ & + & + & 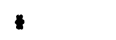 & IMCL & INCL & IMCL \\
\hline 55 & MOVA BIOWED $4+4$ & W/AN & 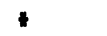 & 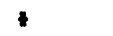 & 1 & 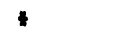 & 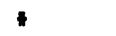 & IMCL & IMCL & IMCL \\
\hline 56 & WONA BIONED $5+5$ & M/AV & + & + & 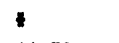 & 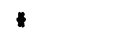 & 1 & IMCL. & INCL & INCL \\
\hline 57 & COBAB FARA & 15.00 & 25.50 & 15.00 & 11.50 & 15.00 & 82.00 & $0.3 \mathrm{~m}$ & VARIES & NO REC CALIB \\
\hline 58 & COEAS MIRA & 11.00 & 18.50 & 11.00 & 8.50 & 11.00 & 60.00 & $0.30 \mathrm{ml}$ & VARIES & WO REC CALIB \\
\hline 59 & ASBIST & $N / D A$ & $M / D A$ & N/DA & N/DA & M/DA & M/DA & N/DA & N/DA & $N / D A$ \\
\hline 60 & CHEN I & 1.24 & 2.30 & 1.15 & 1.24 & 1.24 & 7.17 & W/DA & N/BA & N/DA \\
\hline 61 & $R A-1000$ & 9.05 & 16.64 & 9.05 & 7.16 & 7.16 & 49.06 & $1.2140 \mathrm{Y}$ & $85{ }^{\circ}$ & 3IM $484 R$ \\
\hline 62 & $R A-2 x$ & 9.05 & 16.64 & 9.05 & 7.16 & 7.16 & 49.06 & $1.2140 Y$ & $85 P$ & 3in. 484 R \\
\hline 63 & $M A-500$ & WO U/E & 16.64 & 9.05 & 7.16 & 7.16 & WO U/E & $1.2140 \mathrm{Y}$ & $85{ }^{\circ}$ & 3in $48 \mathrm{n}$ \\
\hline 64 & $R A-X T$ & 9.05 & 16.64 & 9.05 & 7.16 & 7.16 & 49.06 & $1.2140 \mathrm{Y}$ & BSP & 3in. 484 R \\
\hline 65 & $\operatorname{manc} 11$ & 46.04 & 46.04 & 46.04 & 46.04 & $\begin{array}{l}46.04 \\
46.04\end{array}$ & $\begin{array}{l}46.04 \\
46.04\end{array}$ & M/DA & M/DA & W/DA \\
\hline $\begin{array}{l}66 \\
67\end{array}$ & SRA 2000 & 46.04 & 46.04 & $\begin{array}{l}46.04 \\
\text { W/AV }\end{array}$ & $\begin{array}{l}46.04 \\
\text { W/AV }\end{array}$ & $\begin{array}{l}46.04 \\
\text { M/AV }\end{array}$ & $\begin{array}{l}46.04 \\
\text { W/AV }\end{array}$ & $\begin{array}{l}\text { W/DA } \\
\text { W/AV }\end{array}$ & $\begin{array}{l}\text { W/BA } \\
\text { W/AV }\end{array}$ & $\begin{array}{l}\text { N/DA } \\
\text { W/AV }\end{array}$ \\
\hline 64 & $\begin{array}{l}\text { GEA PROFILER } \\
\text { ULTROLAS I }\end{array}$ & WO U/E & 3.00 & 3.00 & 3.00 & 3.00 & W/DA & N/DA & $\mathrm{N} / \mathrm{DA}$ & N/DA \\
\hline 69 & VITALAB 200 & N/OA & M/DA & N/DA & M/OA & M/DA & M/DA & 1.010 & M/DA & 5im \\
\hline
\end{tabular}


No INST ABBOTT UPS
PARALLEL
PERSPECTIU

4 ENCORE II

\section{SPIRIT}

HITACHI 704

HITACHI 705

HITACHI 737

EASY

10 EPOS

12 EXSEL

13 EXTRA

14 AU 5021

15 AU 5121

17. AU 5131

18 AU 5041

19 AU 5141

20 AU 5061

21 AU 5081

22 AS4

23 ASB

24 as ENZYME

25 AS LIPID

26 AS MINI IDEAL

27 AS IDEAL

$28 \mathrm{CXJ}$

29 CX4

30 CX5

31 $\quad \times 7$

32 ASCA

33 CHEMILYSER

34 SYSTEN4

35 PRISMA II

36 CORNING 580

CORNING 570 N/DA

CORNING EXPRESSN/AV

$39 \mathrm{CPA}$

40 DACOS 2

41 DIMENSION 380

42 DIMENSION 76015

43 GREINER 6-400

45 TAMDEM 2

46 TANDEN 3
47 GENESIS 21

48 MONARCH

49 MONARCH PLUS 2

50 EXTACHEN DT 127

51 EKTACHEN $700 \quad 129$

$\begin{array}{lll}52 & \text { PROBRESS } & 140 \\ 53 & \text { SPECIFIC } & \text { N/DA }\end{array}$

54 MOVA $1+1$

55 NOVA 4+4

56 NOVA $5+5$

57 COBAS FARA

58 COBAS MIRA

59 ASSIST

60 CHEN 1

6) RA-1000

62 RA-2X

63 RA 500

$64 R A-X T$

65 SMAC II

66 SRA 2000

67 GEN-PROFIL

68 ULTROLAB I

9 VITALAB 200

N/DA

$N / D A$

$N / D A$

N/DA

156
$N / O A$

N/DA

N/DA

149
$N / D A$

N/AV

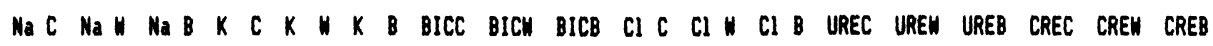

N/AN N/AV N/AV

$\begin{array}{lllllll}143 & 0.8 & 1.7 & 4.7 & 1.2 & 2.0 & \mathrm{~N} / \mathrm{DA}\end{array}$

$\begin{array}{lllllll}143 & 0.8 & 1.7 & 4.7 & 1.2 & 2.0 & \mathrm{~N} / \mathrm{DA}\end{array}$

N/AV N/AV

$\begin{array}{rrrrrrr}145 & 0.5 & N / D A & 4.1 & 1.2 & N / D A & 28.6 \\ 141 & 0.6 & 0.9 & 5.0 & 0.7 & 1.0 & N / D A\end{array}$

$\begin{array}{lllllll}144 & 1.1 & 0.9 & 4.3 & 1.2 & 1.3 & \text { N/DA }\end{array}$

$\begin{array}{lllllll}145 & 0.6 & 0.7 & 5.0 & 0.7 & 1.0 & N / D A\end{array}$

N/DA

\begin{tabular}{ccccccc} 
N/AV & \multicolumn{4}{c}{ N/AV } & & N/AV \\
177 & 0.9 & N/DA & 4.5 & 0.9 & N/DA & 18.0
\end{tabular}

$\begin{array}{llllllllll}177 & 0.9 & \text { N/DA } & 4.5 & 0.9 & \text { N/DA } & 18.0 & 2.1 & \text { N/DA } \\ 117 & 0.9 & \text { NDA } & 4.5 & 0.9 & \text { N/DA } & 18.0 & 2.1 & \text { N/DA }\end{array}$

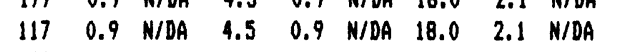

$N / D A$

$N / D A$

N/DA

$\begin{array}{lllll}156 & 0.6 & 0.6 & 5.5 & 1.8 \\ 156 & 0.6 & 0.6 & 5.5 & 1.8\end{array}$

$\begin{array}{ccccc}5.5 & 1.8 & 1.6 & 27.0 \\ 0.6 & 5.6 & 1.8 & \end{array}$

$\begin{array}{lllll}156 & 0.6 & 0.6 & 5.5 & 1.8\end{array}$

$\begin{array}{llll}5.5 & 1.8 & 1.6 & 27.0\end{array}$

N/DA

N/DA

N/AV

139 $1.0 \quad 1.2$ N/AV

$\begin{array}{llllllll}1.2 & 4.9 & 0.8 & 1.0 & 27.0 & 1.6 & 2.2 & \text { N/DA }\end{array}$

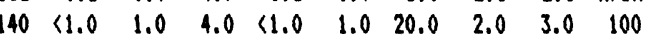

$\begin{array}{lllllll}38 & 0.5 & 0.6 & 4.1 & 0.7 & 0.5 & \text { N/DA }\end{array}$

IDA

N/AV

\begin{tabular}{lll}
1.5 & N/AV \\
\hline & 0.4 & 0
\end{tabular}

101
$N / D A$
$N / D A$

N/DA

$\begin{array}{llll}22.0 & 2.3 & 4.6 & 99\end{array}$

$\begin{array}{lllllll}1 & 1.7 & 2.5 & 4.3 & 2.0 & 2.3 & \mathrm{~N} / \mathrm{DA}\end{array}$

$\begin{array}{llllll}1.7 & 2.5 & 4.3 & 2.0 & 2.3 & \text { N/DA }\end{array}$

$\begin{array}{llllllllll}38 & 0.5 & 0.8 & 4.3 & 1.0 & 1.6 & 27.3 & 3.1 & \mathrm{~N} / \mathrm{DA} & 101\end{array}$

$\begin{array}{llllllllll}138 & 0.5 & 0.8 & 4.3 & 1.0 & 1.6 & 27.3 & 3.1 & \text { N/DA } & 101\end{array}$

$\begin{array}{lllllll}138 & 0.5 & 0.8 & 4.3 & 1.0 & 1.6 & 27.3\end{array}$

$\begin{array}{lllllll}138 & 0.5 & 0.8 & 4.3 & 1.0 & 1.6 & 27.3\end{array}$

$\begin{array}{llllllllll}141 & 0.6 & 1.1 & 4.1 & 1.3 & 1.3 & 27.0 & 2.4 & 6.2 & 96\end{array}$

$\begin{array}{llllllllll}39 & 0.5 & 1.2 & 4.3 & 0.2 & 0.9 & 25.0 & 1.6 & 5.9 & 99\end{array}$

$\begin{array}{llllllllll}39 & 0.5 & 1.2 & 1.3 & 0.7 & 0.9 & 25.0 & 1.6 & 5.9 & 99\end{array}$

$\begin{array}{lllllllll}1.1 & 1.1 & 5.9 & 1.4 & 1.5 & 23.0 & 4.8 & 4.9 & 108 \\ 0.7 & 1.1 & 4.4 & 1.4 & 1.6 & 24.1 & 4.2 & 4.2 & 101\end{array}$

$\begin{array}{lllllll}40 & 0.4 & 0.8 & 4.6 & 1.4 & 1.3 & \mathrm{~N} / \mathrm{DA}\end{array}$

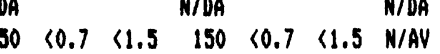

$150<0.7<1.5 \quad 150<0.7<1.5 \quad 22.0$

$150<0.7<1.5 \quad 150<0.7<1.5 \mathrm{~N} / \mathrm{AV}$

$140 \quad 0.5 \quad \mathrm{~N} / \mathrm{DA} \quad 5.0 \quad 0.5 \quad \mathrm{~N} / \mathrm{DA} \quad \mathrm{N} / \mathrm{DA}$

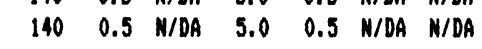

N/DA N/DA

N/DA

$\begin{array}{llllllllll}130 & 0.6 & \text { N/DA } & 4.5 & 1.1 & \text { N/DA } & 25.0 & 2.4 & \text { N/DA } & 103\end{array}$

130
$N / D A$

N/DA

N/AV

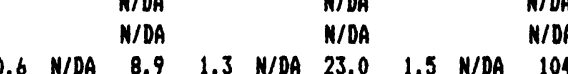

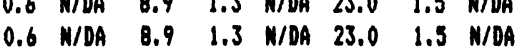

8.9
W/DA

N/AV

N/AV

$N / D A$

N/AV $\begin{array}{llllll}7.6 & 2.9 & 3.4 & 271 & 3.0 & 1.3\end{array}$

$\begin{array}{llllll}10.0 & 1.3 & 2.9 & 340 & 1.5 & 3.0\end{array}$

$\begin{array}{llllll}10.0 & 1.3 & 2.9 & 340 & 1.5 & 3.0\end{array}$

$\begin{array}{llllll}6.7 & 2.0 & 3.5 & 106 & 2.8 & 4.1\end{array}$

$\begin{array}{llllllll}1.3 & 3.0 & 5.0 & 1.3 & 2.2 & 159 & 2.6 & 6.1\end{array}$

$\begin{array}{llllllll}0.6 & 1.1 & 8.3 & 1.1 & 1.7 & 300 & 1.4 & 1.9\end{array}$

$\begin{array}{llllll}26.0 & 1.0 & 1.3 & 336 & 1.5 & 2.1\end{array}$

$\begin{array}{llllllll}0.6 & 1.1 & 15.5 & 1.2 & 1.6 & 404 & 0.9 & 1.4\end{array}$ N/DA N/DA

$8.9 \quad 1.1 \quad \mathrm{~N} / \mathrm{DA} \quad 119 \quad 0.6 \quad \mathrm{~N} / \mathrm{DA}$

$\begin{array}{llllllll}0.5 & \text { N/DA } & 8.0 & 1.1 & \text { N/DA } & 107 & 1.1 & \text { N/DA }\end{array}$

$\begin{array}{llllllll}0.5 & \mathrm{~N} / \mathrm{DA} & 8.0 & 1.1 & \mathrm{~N} / \mathrm{DA} & 107 & 1.1 & \mathrm{~N} / \mathrm{DA}\end{array}$

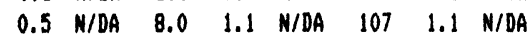

N/DA N/DA

N/DA $\quad$ N/DA

N/DA N/DA

N/DA N/DA

N/DA N/DA

N/DA N/DA

$\begin{array}{llllll}16.8 & 1.5 & 2.6 & 327 & 2.7 & 1.9\end{array}$

$\begin{array}{llllllll}1.4 & 0.5 & 16.8 & 1.5 & 2.6 & 327 & 2.7 & 1.9 \\ 1.4 & 0.5 & 16.8 & 1.5 & 2.6 & 327 & 2.7 & 1.9\end{array}$

$\begin{array}{llllllll}1.4 & 0.5 & 16.8 & 1.5 & 2.6 & 327 & 2.7 & 1.9\end{array}$

$\begin{array}{llllllll}1.4 & 0.5 & 16.8 & 1.5 & 2.6 & 327 & 2.7 & 1.9\end{array}$

$\begin{array}{llllllll}1.4 & 0.5 & 16.8 & 1.5 & 2.6 & 327 & 2.7 & 1.9\end{array}$

$\begin{array}{lllllllll}1.4 & 0.5 & 16.8 & 1.5 & 2.6 & 327 & 2.7 & 1.9\end{array}$

$N / D A \quad N / D A$

N/DA N/DA

$N / D A \quad N / D A$

N/DA N/DA

N/DA N/DA

$\begin{array}{llllll}21.5 & 0.6 & 1.9 & 375 & 1.5 & 1.6\end{array}$

$\begin{array}{llllll}8.8 & 1.7 & 1.9 & 143 & 0.9 & 1.0\end{array}$

$\begin{array}{llllllll}1.0 & 2.0 & 7.0 & 1.0 & 2.0 & 80 & 2.0 & 3.0\end{array}$

$\begin{array}{llllllll}0.4 & 0.6 & 4.0 & 3.1 & 1.4 & 237 & 1.0 & 1.0\end{array}$

$\begin{array}{llllll}N / D A & 2.0 & 3.0 & \text { N/DA } & 2.5 & 3.0\end{array}$

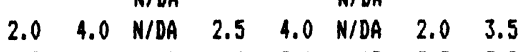

$\begin{array}{lllllllll}106 & 0.5 & 2.6 & 6.9 & 1.4 & 2.6 & 112 & 2.7 & 3.7\end{array}$

$\begin{array}{lllllllll}106 & 1.1 & 1.9 & 2.2 & 2.6 & 4.8 & 236 & 1.8 & 3.4\end{array}$

$\begin{array}{lllllllll}0.9 & 1.5 & 16.5 & 0.9 & 1.7 & 345 & 0.4 & 1.4\end{array}$

$\begin{array}{llllllll}0.9 & 1.5 & 16.5 & 0.9 & 1.7 & 345 & 0.4 & 1.4\end{array}$

$\begin{array}{llllllll}0.9 & 1.5 & 16.5 & 0.9 & 1.7 & 345 & 0.4 & 1.4\end{array}$

$\begin{array}{llllllll}0.9 & 1.5 & 16.5 & 0.9 & 1.7 & 345 & 0.4 & 1.4\end{array}$

$\begin{array}{llllllll}1.0 & 1.5 & 15.6 & 2.1 & 2.6 & 88 & 2.2 & 4.4\end{array}$

$\begin{array}{llllllll}0.4 & 1.1 & 18.2 & 1.4 & 2.9 & 102 & 2.0 & 3.3\end{array}$

$\begin{array}{llllllll}0.4 & 1.1 & 18.2 & 1.4 & 2.9 & 102 & 2.0 & 3.3\end{array}$

$\begin{array}{llllllll}1.0 & 1.5 & 7.8 & 2.4 & 2.6 & 185 & 5.3 & 5.7\end{array}$

$\begin{array}{rrrrrrrrr}\text { N/DA } & 0.7 & 0.9 & 12.3 & 1.9 & 2.8 & 521 & 1.0 & 1.7 \\ \text { N/DA } & & & 8.1 & 1.3 & 2.5 & 120 & 1.5 & 3.0\end{array}$

N/DA 2.5 N/DA

N/AV N/AV

$175<0.7<1.5 \mathrm{~N} / \mathrm{AV} \quad \mathrm{N} / \mathrm{AV}$

$160<0.7<1.5 \mathrm{~N} / \mathrm{AV} \quad \mathrm{N} / \mathrm{AV}$

$\begin{array}{lllllll}\text { N/DA } & 4.9 & 1.8 & 2.1 & 141 & 1.9 & 1.3\end{array}$

$\begin{array}{lll}N / D A & N / D A & N / D A \\ N / D A & N / D A & N / D A\end{array}$

$7.1 \quad \mathrm{~N} / \mathrm{DA} \quad 1.7 \quad \mathrm{~N} / \mathrm{DA}$

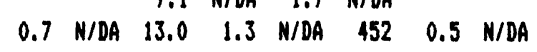

0.7 N/DA $13.0 \quad 1.3 \quad \mathrm{~N} / \mathrm{DA} \quad 452 \quad 0.5 \mathrm{~N} / \mathrm{DA}$

$\begin{array}{ll}\text { N/DA } & \text { N/DA } \\ \text { N/DA } & \text { N/DA }\end{array}$

$\begin{array}{lllllllll}104 & 0.9 & \text { N/DA } & 10.5 & 1.2 & \text { N/DA } & 340 & 1.6 & \text { N/DA }\end{array}$

$104 \begin{array}{llllllll}0.9 & \text { N/DA } & 10.5 & 1.2 & \text { N/DA } & 340 & 1.6 & \text { N/DA }\end{array}$

W/DA $\quad$ N/DA

$\begin{array}{llll}\text { N/AV } & \text { N/DA } & \text { N/DA } & \text { N/DA } \\ \text { N/DA } & \text { N/DA } & \text { N/DA } & \text { N/DA }\end{array}$ 


\begin{tabular}{|c|c|c|c|c|c|c|c|c|c|c|c|c|c|c|c|c|c|c|c|}
\hline No & INST & TP C & TP N & TP B & ALBC & ALBW & ALBB & BILC & BILW & BILB & ALPC & ALPW & ALPB & $\mathrm{HBDC}$ & HBOW $\mathrm{H}$ & HBDB & ASTC & ASTW & ASTB \\
\hline 1 & ABBOTT UPS & 68 & 1.0 & 0.8 & 42 & 0.8 & 0.9 & 84 & 1.6 & 2.3 & 218 & 1.7 & 3.8 & 249 & 1.3 & 2.1 & 110 & 1.3 & 2.9 \\
\hline 2 & PARALLEL & 60 & 1.0 & 2.0 & 45 & 1.5 & 3.0 & 70 & 1.0 & 1.7 & 150 & 2.0 & 4.4 & N/AV & & & 80 & 2.5 & 4.9 \\
\hline 3 & PERSPECTIVE & 60 & 1.0 & 2.0 & 45 & 1.5 & 3.0 & 70 & 1.0 & 1.7 & 150 & 2.0 & 4.4 & N/AV & & & 80 & 2.5 & 4.9 \\
\hline 4 & ENCORE II & 60 & 1.2 & 2.7 & 46 & 1.2 & 2.4 & 104 & 1.1 & 2.5 & 157 & 1.0 & 2.1 & 170 & 1.2 & 2.1 & 83 & 1.2 & 4.5 \\
\hline 5 & SPIRIT & 53 & 1.3 & 3.7 & 37 & 1.1 & 2.7 & 15 & 3.3 & 5.0 & 98 & 1.1 & 4.5 & N/AV & & & 43 & 2.4 & 5.6 \\
\hline 6 & HITACHI 704 & 55 & 0.8 & 1.0 & 36 & 1.0 & 1.2 & 39 & 0.5 & 1.3 & 490 & 0.8 & 1.5 & N/DA & & & 100 & 0.7 & 1.1 \\
\hline 7 & HITACHI 705 & 60 & 1.9 & 3.0 & 37 & 1.1 & 1.5 & 7.1 & 0.6 & 1.4 & 1235 & 0.6 & 1.4 & 250 & 1.2 & 2.2 & 471 & 1.0 & 2.0 \\
\hline 8 & HITACHI 737 & 63 & 0.8 & 1.2 & 42 & 1.1 & 2.1 & 74 & 0.8 & 1.3 & 504 & 0.8 & 1.1 & N/DA & & & 105 & 0.7 & 1.1 \\
\hline & EASY & $N / D A$ & & & $M / D A$ & & & N/DA & & & $N / D A$ & & & N/DA & & & $N / D A$ & & \\
\hline 10 & EPOS & 44 & 1.0 & N/DA & 21 & 0.8 & $N / D A$ & 20 & 2.7 & N/DA & 257 & 1.4 & N/DA & 69 & 1.2 & N/DA & 27 & 1.6 & N/DA \\
\hline 11 & ERIS & 69 & 0.8 & $N / D A$ & 26 & 1.1 & $N / D A$ & 20 & 1.1 & $N / D A$ & 286 & 0.7 & $N / D A$ & 233 & 1.2 & N/DA & 44 & 1.1 & N/DA \\
\hline 12 & EXSEL & 69 & 0.8 & N/DA & 26 & 1.1 & $N / D A$ & 20 & 1.1 & $N / D A$ & 286 & 0.7 & $N / D A$ & 233 & 1.2 & $N / D A$ & 44 & 1.1 & $N / D A$ \\
\hline 13 & EXTRA & 69 & 0.8 & $N / D A$ & 26 & 1.1 & $N / D A$ & 20 & 1.1 & $N / D A$ & 286 & 0.7 & $N / D A$ & 233 & 1.2 & N/DA & 44 & 1.1 & N/DA \\
\hline 14 & AU 5021 & $N / D A$ & & & $N / D A$ & & & N/DA & & & $N / D A$ & & & N/DA & & & N/DA & & \\
\hline 15 & AU 5121 & $N / D A$ & & & $N / D A$ & & & $N / D A$ & & & $N / D A$ & & & N/DA & & & $N / D A$ & & \\
\hline 16 & AU 5031 & $N / D A$ & & & N/DA & & & $N / D A$ & & & $N / D A$ & & & $N / D A$ & & & N/DA & & \\
\hline 17 & AU 5131 & N/DA & & & $N / D A$ & & & $N / D A$ & & & $N / D A$ & & & $N / D A$ & & & N/DA & & \\
\hline 18 & AU 5041 & $N / D A$ & & & $N / D A$ & & & $N / D A$ & & & $N / D A$ & & & $N / D A$ & & & $N / D A$ & & \\
\hline 19 & AU 5141 & N/DA & & & $N / D A$ & & & $N / D A$ & & & $N / D A$ & & & $N / D A$ & & & $N / D A$ & & \\
\hline 20 & AU 5061 & $N / D A$ & & & $N / D A$ & & & $N / D A$ & & & $N / D A$ & & & $N / D A$ & & & $N / D A$ & & \\
\hline 21 & AU 5081 & $N / D A$ & & & $N / D A$ & & & $N / D A$ & & & N/DA & & & $N / D A$ & & & $N / D A$ & & \\
\hline 22 & AS4 & 71 & 2.4 & 2.9 & 48 & 0.7 & 1.2 & 84 & 3.4 & 7.0 & N/AV & & & N/AV & & & N/AV & & \\
\hline 23 & A58 & 71 & 2.4 & 2.9 & 48 & 0.7 & 1.2 & 84 & 3.4 & 7.0 & 146 & 1.4 & 2.5 & N/AV & & & 97 & 3.0 & 2.7 \\
\hline 24 & AS ENZYME & 71 & 2.4 & 2.9 & 48 & 0.7 & 1.2 & 84 & 3.4 & 7.0 & 146 & 1.4 & 2.5 & N/AV & & & 97 & 3.0 & 2.7 \\
\hline 25 & AS LIPID & 71 & 2.4 & 2.9 & 48 & 0.7 & 1.2 & 84 & 3.4 & 7.0 & 146 & 1.4 & 2.5 & N/AV & & & 97 & 3.0 & 2.7 \\
\hline 26 & AS MINI IDEAL & 71 & 2.4 & 2.9 & 48 & 0.7 & 1.2 & 84 & 3.4 & 7.0 & 146 & 1.4 & 2.5 & N/AV & & & 97 & 3.0 & 2.7 \\
\hline 27 & AS IDEAL & 71 & 2.4 & 2.9 & 48 & 0.7 & 1.2 & 84 & 3.4 & 7.0 & 146 & 1.4 & 2.5 & N/AV & & & 97 & 3.0 & 2.7 \\
\hline 28 & $c \times 3$ & $N / D A$ & & & $N / D A$ & & & N/DA & & & $N / D A$ & & & $N / D A$ & & & $N / D A$ & & \\
\hline 29 & $C \times 4$ & $N / D A$ & & & $N / D A$ & & & $N / D A$ & & & $N / D A$ & & & $N / D A$ & & & $N / D A$ & & \\
\hline 30 & Cx5 & $N / D A$ & & & $N / D A$ & & & $N / D A$ & & & $N / D A$ & & & $N / D A$ & & & $N / D A$ & & \\
\hline 31 & $\mathrm{CX7}$ & N/DA & & & $M / D A$ & & & $N / D A$ & & & $N / D A$ & & & $N / D A$ & & & $N / D A$ & & \\
\hline 32 & ASCA & $N / D A$ & & & $N / D A$ & & & $N / D A$ & & & $N / D A$ & & & $N / D A$ & & & $N / D A$ & & \\
\hline 33 & CHEMILYSER & 65 & 1.0 & 1.2 & 32 & 1.3 & 1.5 & 23 & 0.9 & 1.3 & 175 & 1.0 & 1.4 & N/AV & & & N/AV & & \\
\hline 34 & SYSTEMA & 72 & 0.8 & 1.0 & 32 & 1.8 & 2.1 & 21 & 1.1 & 1.4 & 196 & 2.0 & 2.2 & N/AV & & & N/AV & & \\
\hline 35 & PRISMA II & 60 & $<1.0$ & 1.0 & 30 & $<1.0$ & 2.0 & 30 & 1.0 & 3.0 & 180 & 2.0 & 4.0 & 150 & 2.0 & 4.0 & 40 & 2.0 & 5.0 \\
\hline 36 & CORNING 580 & 46 & 0.7 & 0.6 & 30 & 1.8 & 1.8 & 9.1 & 1.2 & 1.2 & 73 & 2.4 & 2.9 & N/DA & & & 22 & 5.5 & 1.9 \\
\hline 37 & CORNING 570 & $N / D A$ & & & $N / D A$ & & & N/DA & & & N/DA & & & $N / D A$ & & & $N / D A$ & & \\
\hline 38 & CORNING EXPRESS & SN/DA & & & $M / D A$ & & & $N / D A$ & & & N/DA & & & $N / D A$ & & & $N / D A$ & & \\
\hline 39 & CPA & $N / D A$ & & & $N / D A$ & & & N/DA & & & N/DA & & & N/DA & & & $N / D A$ & & \\
\hline 40 & DACOS 2 & 62 & 0.7 & 1.4 & 36 & 1.1 & 1.5 & 35 & 2.8 & 4.3 & 84 & 1.8 & 3.6 & N/DA & & & 70 & 2.1 & 3.3 \\
\hline 41 & DIMENSION 380 & 67 & 1.5 & 2.0 & 37 & 2.9 & 2.9 & 32 & 0.9 & 3.4 & N/DA & & & N/AV & & & 186 & 3.0 & 4.4 \\
\hline 42 & DIMENSIOM 760 & 67 & 1.5 & 2.0 & 37 & 2.9 & 2.9 & 32 & 0.9 & 3.4 & N/DA & & & N/AV & & & 186 & 3.0 & 4.4 \\
\hline 43 & GREIMER G-400 & 71 & 0.5 & 1.5 & 39 & 0.6 & 1.8 & 83 & 0.4 & 1.4 & 217 & 1.1 & 2.4 & 581 & 2.6 & 3.2 & 149 & 1.5 & 2.5 \\
\hline 44 & GREIMER 6-450 & 71 & 0.5 & 1.5 & 39 & 0.6 & 1.8 & 83 & 0.4 & 1.4 & 217 & 1.1 & 2.4 & 581 & 2.6 & 3.2 & 149 & 1.5 & 2.5 \\
\hline 45 & TANDEM 2 & 71 & 0.5 & 1.5 & 39 & 0.6 & 1.8 & 83 & 0.4 & 1.4 & 217 & 1.1 & 2.4 & 518 & 2.6 & 3.2 & 149 & 1.5 & 2.5 \\
\hline 46 & TAMDEM 3 & 71 & 0.5 & 1.5 & 39 & 0.6 & 1.8 & 83 & 0.4 & 1.4 & 217 & 1.1 & 2.4 & 581 & 2.6 & 3.2 & 149 & 1.5 & 2.5 \\
\hline 47 & GENESIS 21 & 60 & 0.8 & 1.7 & 38 & 1.1 & 2.4 & 5 & 1.3 & 2.1 & 126 & 1.7 & 3.5 & 480 & 1.6 & 2.1 & 90 & 0.7 & 1.3 \\
\hline 48 & MOMARCH & 68 & 1.2 & 2.5 & 38 & 0.6 & 3.0 & 65 & 1.5 & 2.5 & 74 & 1.3 & 3.3 & 370 & 1.6 & 4.3 & 75 & 1.1 & 2.4 \\
\hline 49 & MONARCH PLUS 2 & 68 & 1.2 & 2.5 & 38 & 0.6 & 3.0 & 65 & 1.5 & 2.5 & 74 & 1.3 & 3.3 & 370 & 1.6 & 4.3 & 75 & 1.1 & 2.4 \\
\hline 50 & EKTACHEM DT & 68 & 2.2 & 2.2 & N/AV & & & 33 & 5.3 & 7.4 & 74 & 2.5 & 4.8 & N/AV & & & 70 & 5.6 & 7.1 \\
\hline $5 !$ & EKTACHEY 700 & 72 & 1.8 & 2.2 & 36 & 1.7 & 1.7 & 68 & 2.0 & 3.2 & 88 & 2.5 & 4.2 & N/AV & & & 202 & 1.2 & 2.8 \\
\hline 52 & PROGRESS & 70 & 0.6 & 1.0 & 34 & 1.0 & 1.3 & 26 & 2.2 & 1.6 & 230 & 2.2 & 2.8 & N/DA & & & 63 & 1.2 & 2.2 \\
\hline 53 & SPECIFIC & N/DA & & & $N / D A$ & & & $N / D A$ & & & $N / D A$ & & & $M / D A$ & & & $N / D A$ & & \\
\hline 54 & NOVA $1+1$ & N/AV & & & N/AV & & & N/AY & & & M/AV & & & N/AV & & & N/AV & & \\
\hline 55 & NOVA $4+4$ & N/AV & & & N/AV & & & N/AV & & & N/AV & & & N/AV & & & N/AV & & \\
\hline 56 & MOVA $5+5$ & N/AV & & & N/AV & & & N/AV & & & N/AV & & & N/AV & & & N/AV & & \\
\hline 57 & COBAS FARA & 56 & 0.8 & 1.2 & 62 & 1.6 & 1.8 & 34 & 0.9 & 2.9 & 114 & 0.4 & 1.2 & 263 & 1.0 & 1.7 & 169 & 1.7 & 2.1 \\
\hline 58 & COBAS MIRA & 72 & 1.2 & 1.7 & 36 & 1.6 & 2.5 & 112 & 1.0 & 1.5 & 182 & 0.6 & 0.9 & 274 & 1.9 & 3.1 & 95 & 0.7 & 1.1 \\
\hline 59 & ASSIST & N/DA & & & N/DA & & & N/DA & & & N/DA & & & N/DA & & & N/DA & & \\
\hline 60 & CHEM 1 & $N / D A$ & & & 35 & N/DA & 1.4 & N/DA & & & 162 & N/DA & 1.6 & N/AV & & & 183 & $M / D A$ & 1.7 \\
\hline 61 & $R A-1000$ & 75 & 1.0 & N/DA & 43 & 1.3 & N/DA & 85 & 2.0 & N/DA & 64 & 0.7 & N/DA & N/AV & & & 300 & 1.3 & N/DA \\
\hline 62 & $R A-2 X$ & 75 & 1.0 & N/DA & 13 & 1.3 & N/DA & 85 & 2.0 & N/DA & 64 & 0.7 & N/DA & N/AV & & & 300 & 1.3 & N/DA \\
\hline 63 & $R A-500$ & $M / D A$ & & & $N / D A$ & & & H/DA & & & N/DA & & & M/DA & & & $N / D A$ & & \\
\hline 64 & $R A-X T$ & $N / D A$ & & & N/DA & & & N/DA & & & $N / D A$ & & & N/DA & & & $N / D A$ & & \\
\hline 65 & SUAC II & 64 & 1.1 & N/DA & 38 & 1.3 & N/DA & 76 & 1.5 & N/BA & 153 & 1.3 & $N / D A$ & N/AV & & & 200 & 1.3 & N/DA \\
\hline 66 & SRA 2000 & 64 & 1.1 & N/DA & 38 & 1.3 & N/DA & 76 & 1.5 & N/DA & 153 & 1.3 & N/DA & N/AV & & & 200 & 1.3 & $\mathrm{~N} / \mathrm{DA}$ \\
\hline 67 & BEH-PROFILER & N/DA & & & $N / D A$ & & & $N / D A$ & & & N/DA & & & M/DA & & & N/DA & & \\
\hline 68 & ULTROLAB I & $N / D A$ & & & $N / D A$ & & & $N / D A$ & & & N/DA & & & $N / D A$ & & & N/DA & & \\
\hline 69 & VITALAB 200 & $M / D A$ & & & $N / D A$ & & & $N / D A$ & & & M/DA & & & N/DA & & & N/DA & & \\
\hline
\end{tabular}


No INST

1 ABBOTT UPS

2 PARALLEL

3 PERSPECTIVE

4 ENCORE II

5 SPIRIT

6 HITACHI 704

7 HITACHI 705

8 HITACHI 737

9 EASY

10 EPOS

11 ERIS

12 EXSEL

13 EXTRA

14 AU 5021

15 AU 5121

16 AU 5031

17 AU 5131

18 AU 5041

19 AU 5141

20 AU 5061

21 AU 5081

22 A54

23 ASB

24 AS ENZYME

25 AS LIPID

26 AS MINI IDEAL

27 AS IDEAL

$28 \mathrm{CXJ}$

$29 \quad \mathrm{CX}$

$30 \mathrm{Cx5}$

$31 \quad \mathrm{CX} 7$

32 ASCA

33 CHEMILYSER

34 SYSTEM4

35 PRISHA II

36580 ALLIANCE

37500

38 CORNING EXPRESS N/DA

39 CPA

40 DACOS 2

41 DIMENSION 380

42 DIMENSION 760

43 6-400

$44 \quad 6-450$

45 TANDEN 2

46 TANDEN 3

47 GEMESIS 21

48 MOMARCH

49 MONARCH PLUS 2

50 EKTACHEN DT

51 EKTACHEM 700

52 PROGRESS

53 SPECIFIC

54 NOVA $1+1$

55 NOVA 4+A

56 MOVA $5+5$

57 COBAS FARA

58 COBAS MIRA

59 ASSIST

60 CHEH 1

6) $R A-1000$

62 RA-2X

$63 \quad R A-500$

64 RA-XT

65 SHAC II

66 SRA 2000

67 GEH-PROFILER

68 ULTROLAB I

69 VITALAB 200

ALTC ALTW ALTB 66TC G6TW 66TB LDHC LDHW LDHB CK C CK W CK B CALC CALN CALB POAC PO4W PO4B

$\begin{array}{lllllllllllllllllll}45 & 1.8 & 2.3 & 73 & 1.0 & 3.1 & 310 & 1.7 & 2.5 & 64 & 2.4 & 4.0 & 2.25 & 1.5 & 1.0 & 1.78 & 1.3 & 2.1\end{array}$ $\begin{array}{llllllllllllllllll}80 & 3.0 & 6.0 & 40 & 3.0 & 8.0 \mathrm{~N} / \mathrm{AV} & \text { ???? } & 2.8 & 5.5 & 2.50 & 1.0 & 1.5 & 1.30 & 1.0 & 1.6\end{array}$ $\begin{array}{llllllllllllllllll}80 & 3.0 & 6.0 & 40 & 3.0 & 8.0 & \text { N/AV } & \text { N/DA } & 2.50 & 1.0 & 1.5 & 1.30 & 1.0 & 1.6\end{array}$

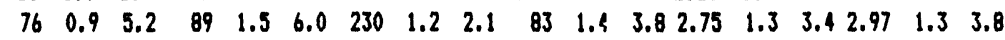

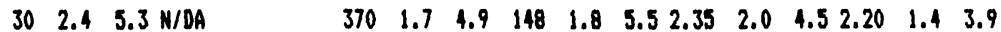
$\begin{array}{lllllllllllllll}55 & 1.3 & 2.2 & 104 & 1.0 & 1.3 & 329 & 1.1 & 1.9 & 360 & 0.7 & 1.5 & 2.30 & 0.9 & 1.4 \\ \text { N/DA }\end{array}$

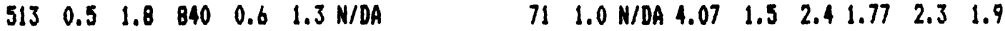
$\begin{array}{llllllllllllllllll}128 & 0.7 & 1.1 & 178 & 0.9 & 1.2 & 498 & 1.2 & 1.5 & 365 & 0.7 & 1.5 & 2.63 & 0.9 & 1.9 & 1.50 & 1.2 & 1.7\end{array}$ N/DA N/DA N/DA $18 \mathrm{~N} / \mathrm{DA} 3.0 \mathrm{~N} / \mathrm{DA} \quad$ N/DA

$\begin{array}{lllllllllllll}30 & 1.9 & \mathrm{~N} / \mathrm{DA} & 32 & 1.5 \mathrm{~N} / \mathrm{DA} & 148 & 1.3 \mathrm{~N} / \mathrm{DA} & 48 & 2.0 \mathrm{~N} / \mathrm{DA} & 1.33 & 0.7 \mathrm{~N} / \mathrm{DA} & 1.64 & 2.3 \mathrm{~N} / \mathrm{DA}\end{array}$

$44 \quad 1.2 \mathrm{~N} / \mathrm{DA} \quad 62 \quad 0.5 \mathrm{~N} / \mathrm{DA} \quad 415 \quad 1.4 \mathrm{~N} / \mathrm{DA} \quad 48 \quad 2.0 \mathrm{~N} / \mathrm{DA} 2.59 \quad 1.1 \mathrm{~N} / \mathrm{DA} \quad 1.12 \quad 1.8 \mathrm{~N} / \mathrm{DA}$

$44 \quad 1.2 \mathrm{~N} / \mathrm{DA} \quad 62 \quad 0.5 \mathrm{~N} / \mathrm{DA} \quad 415 \quad 1.4 \mathrm{~N} / \mathrm{DA} \quad 48 \quad 2.0 \mathrm{~N} / \mathrm{DA} \quad 2.59 \quad 1.1 \mathrm{~N} / \mathrm{DA} \quad 1.12 \quad 1.8 \mathrm{~N} / \mathrm{DA}$ $44 \quad 1.2 \mathrm{~N} / \mathrm{DA} \quad 62 \quad 0.5 \mathrm{~N} / \mathrm{DA} \quad 415 \quad 1.4 \mathrm{~N} / \mathrm{DA} \quad 48 \quad 2.0 \mathrm{~N} / \mathrm{DA} 2.59 \quad 1.1 \mathrm{~N} / \mathrm{DA} \quad 1.12 \quad 1.8 \mathrm{~N} / \mathrm{DA}$

N/DA N/DA N/DA N/DA N/DA N/DA

N/DA N/DA N/DA $\quad N / D A \quad N / D A \quad N / D A$

N/DA N/DA N/DA N/DA N/DA N/DA

N/DA N/DA N/DA N/DA N/DA N/DA

N/DA N/DA N/DA N/DA N/DA N/DA

N/DA N/DA N/DA N/DA N/DA N/DA

N/DA N/DA N/DA N/DA N/DA N/DA

N/DA N/DA N/DA N/DA N/DA N/DA

N/AV N/AV N/AV N/AV $3.301 .3 \quad 1.51 .941 .5$ N/DA

$\begin{array}{lllllllllllllllll}95 & 3.2 & 3.7 & 110 & 2.7 & 3.9 & 306 & 1.6 & 2.7 & 148 & 2.0 & 2.3 & 3.30 & 1.3 & 1.5 & 1.94 & 1.5\end{array}$ N/DA $\begin{array}{lllllllllllllllll}95 & 3.2 & 3.7 & 110 & 2.7 & 3.9 & 306 & 1.6 & 2.7 & 148 & 2.0 & 2.3 & 3.30 & 1.3 & 1.5 & 1.94 & 1.5\end{array}$ $\begin{array}{lllllllllllllllll}95 & 3.2 & 3.7 & 110 & 2.7 & 3.9 & 306 & 1.6 & 2.7 & 148 & 2.0 & 2.3 & 3.30 & 1.3 & 1.5 & 1.94 & 1.5\end{array}$ $\begin{array}{lllllllllllllllll}95 & 3.2 & 3.7 & 110 & 2.7 & 3.9 & 306 & 1.6 & 2.7 & 148 & 2.0 & 2.3 & 3.30 & 1.3 & 1.5 & 1.94 & 1.5\end{array}$ $\begin{array}{lllllllllllllllll}95 & 3.2 & 3.7 & 110 & 2.7 & 3.9 & 306 & 1.6 & 2.7 & 148 & 2.0 & 2.3 & 3.30 & 1.3 & 1.5 & 1.94 & 1.5\end{array}$ N/DA N/DA $\quad$ N/DA $\quad$ N/DA $\quad$ N/DA N N/DA N/DA N/DA N/DA N/DA N/DA N/DA N/DA N/DA N/DA N/DA N/DA N/DA N/DA N/DA N/DA N/DA N/DA N/DA

N/DA N/DA N/DA N/DA N/DA N/DA

$\begin{array}{llllllllllllll}\text { N/AV } & 56 & 1.5 & 1.5 \mathrm{~N} / \mathrm{AV} & \text { N/DA } & 2.40 & 1.3 & 1.5 & 1.05 & 0.8 & 1.0\end{array}$

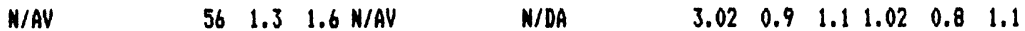
$\begin{array}{lllllllllllllllllll}40 & 2.0 & 5.0 & 60 & 2.0 & 5.0 & 300 & 2.0 & 4.0 & 150 & 2.5 & 3.0 & 2.00<1.0 & 2.0 & 1.00 & 1.0 & 3.0\end{array}$ $\begin{array}{llllllllllllllllll}18 & 9.6 & 5.1 & 18 & 4.8 & 2.4 & 120 & 3.2 & 1.4 & 135 & 1.5 & 1.6 & 1.86 & 0.7 & 1.1 & N / D A & 1.0 & 2.0\end{array}$ N/DA N/DA N/DA N/DA N/DA N/DA

N/DA N/DA N/DA N/DA N/DA N/DA

N/DA N/DA N/DA N/DA N/DA N/DA

$\begin{array}{llllllllllllllllll}108 & 1.6 & 4.6 & 152 & 1.1 & 2.3 & 682 & 0.8 & 1.0 & 177 & 1.2 & 2.0 & 2.60 & 0.9 & 2.4 & 1.26 & 0.8 & 1.7\end{array}$

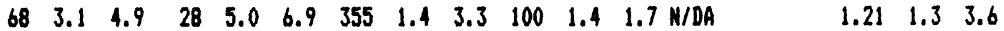
$\begin{array}{lllllllllllllllll}68 & 3.1 & 4.9 & 28 & 5.0 & 6.9 & 355 & 1.4 & 3.3 & 100 & 1.4 & 1.7 & \mathrm{~N} / \mathrm{DA} & 1.21 & 1.3 & 3.6\end{array}$ $\begin{array}{llllllllllllllllll}59 & 2.8 & 4.9 & 140 & 0.9 & 1.6 & 240 & 1.5 & 2.0 & 275 & 1.0 & 5.1 & 2.62 & 0.3 & 1.7 & 1.03 & 0.8 & 2.4\end{array}$ $\begin{array}{llllllllllllllllll}59 & 2.8 & 4.9 & 140 & 0.9 & 1.6 & 240 & 1.5 & 2.0 & 275 & 1.0 & 5.1 & 2.62 & 0.3 & 1.7 & 1.03 & 0.8 & 2.4\end{array}$ $\begin{array}{lllllllllllllllllll}59 & 2.8 & 4.9 & 140 & 0.9 & 1.6 & 240 & 1.5 & 2.0 & 275 & 1.0 & 5.1 & 2.62 & 0.3 & 1.7 & 1.03 & 0.8 & 2.4\end{array}$ $\begin{array}{llllllllllllllllll}59 & 2.8 & 4.9 & 140 & 0.9 & 1.6 & 240 & 1.5 & 2.0 & 275 & 1.0 & 5.1 & 2.62 & 0.3 & 1.7 & 1.03 & 0.8 & 2.4\end{array}$ $\begin{array}{llllllllllllllllll}89 & 0.7 & 1.3 & 63 & 1.3 & 2.9 & 480 & 1.6 & 2.1 & 94 & 1.7 & 3.4 & 2.24 & 0.9 & 2.0 & 0.65 & 0.9 & 1.6\end{array}$ $\begin{array}{llllllllllllllllll}76 & 1.2 & 2.3 & 69 & 0.9 & 2.5 & 380 & 1.7 & 4.2 & 121 & 1.3 & 3.1 & 2.42 & 1.4 & 1.7 & 1.17 & 1.4 & 2.0\end{array}$ $\begin{array}{llllllllllllllllll}76 & 1.2 & 2.3 & 69 & 0.9 & 2.5 & 380 & 1.7 & 4.2 & 121 & 1.3 & 3.1 & 2.42 & 1.4 & 1.7 & 1.17 & 1.4 & 2.0\end{array}$ $\begin{array}{llllllllllllll}71 & 4.3 & 4.6 & 90 & 2.2 & 2.3 & 649 & 0.8 & 2.9 & 175 & 1.8 & 3.0 & \text { N/AV N/AV }\end{array}$

$\begin{array}{llllllllllllllllll}184 & 1.0 & 2.8 & 89 & 1.7 & 2.7 & 489 & 2.6 & 3.2 & 108 & 4.1 & 8.2 & 2.35 & 0.9 & 1.3 & 1.08 & 2.1 & 2.1\end{array}$

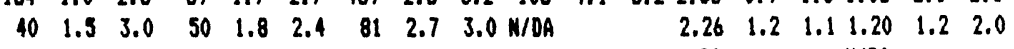
N/DA N/DA N/DA N/DA N/DA N/DA W/AV N/AV N/AV N/AV N/AV N/AV N/AV N/AV N/AV N/AV N/AV N/AV

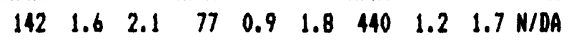
$\begin{array}{llllllllll}97 & 0.5 & 0.8 & 92 & 0.9 & 1.5 & 271 & 1.9 & 3.1 & \mathrm{~N} / \mathrm{DA}\end{array}$ N/DA N/DA N/DA N/DA N/DA N/DA $\quad 130 \mathrm{~N} / \mathrm{DA} \quad 1.6 \mathrm{~N} / \mathrm{DA}$

$300 \mathrm{~N} / \mathrm{DA} \quad 1.3 \quad 108 \mathrm{~N} / \mathrm{DA} \quad 1.0 \mathrm{~N} / \mathrm{AV} \quad \mathrm{N} / \mathrm{DA}$

$300 \mathrm{~N} / \mathrm{DA} \quad 1.3 \quad 108 \mathrm{~N} / \mathrm{DA} \quad 1.0 \mathrm{~N} / \mathrm{AV}$ N/DA N/DA N/DA N/DA N/DA N/DA N/DA N/DA N/DA

$38 \quad 3.8$ N/DA $65 \quad 4.3 \mathrm{~N} / \mathrm{DA} \quad 422 \quad 2.3 \mathrm{~N} / \mathrm{DA} \mathrm{N} / \mathrm{DA}$ $383.8 \mathrm{~N} / \mathrm{DA} \quad 65 \quad 4.3 \mathrm{~N} / \mathrm{DA} 422 \quad 2.3 \mathrm{~N} / \mathrm{DA} \mathrm{N} / \mathrm{DA}$ $\begin{array}{cccc}\text { N/DA } & \text { N/DA } & \text { N/DA } & \text { N/DA } \\ \text { N/DA } & N / D A & N / D A & N / D A \\ N / D A & N / D A & N / D A & N / D A\end{array}$

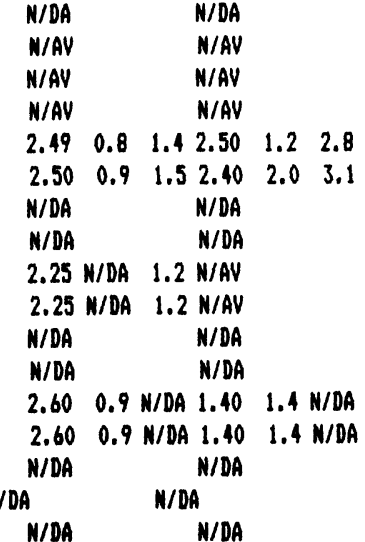


INST

1 ABBOTT UPS

2 PARALLEL

3 PERSPECTIVE

4 ENCORE II

5 SPIRIT

6 HITACHI 704

7 HITACHI 705

8 HITACHI 737

9 EASY

10 EPOS

11 ERIS

12 EXSEL

13 EXTRA

14 AU 5021

15 AU 5121

16 AU 5031

17 AU 5131

18 AU 5041

19 AU 5141

20 AU 5061

21 AU 5081

22 A54

23 ASB

24 AS ENZYME

25 AS LIPID

26 AS MINI IDEAL

27 AS IDEAL

$28 \mathrm{CX}$

$29 \quad \mathrm{CX} 4$

$30 \quad \mathrm{Cx} 5$

$31 \quad \mathrm{CX} 7$

32 ASCA

33 CHEMILYSER

34 SYSTEMA

35 PRISHA II

36 CORNING 580

37 CORNING 570

38 CORMING EXPRESS N/DA

39 CPA

40 DACOS 2

41 DIMENSION 380

42 DIMENSION 760

43 6-400

$14 \quad 6-450$

45 TANDEN 2

46 TAMDEN 3

47 GENESIS 21

48 MONARCH

49 MOMARCH PLUS 2

50 EXTACHEN DT

51 EKTACHEN 700

52 PROGRESS

53 SPECIFIC

54 NOVA $1+1$

55 MOVA $1+4$

56 NOVA $5+5$

57 COBAS FARA

58 COBAS MIRA

59 ASSIST

60 CHEN 1

61 RA-1000

62 RA-2X

63 $R A-500$

$64 R A-X T$

65 SHAC II

66 SRA 2000

67 GEN-PROFILER

68 ULTROLAB I

69 VITALAB 200
CHOC CHOW CHOB tG C TG N TG B URAC URAM URAB GLUC GLUW GLUB

$\begin{array}{llllllllllll}2.3 & 1.3 & 2.7 & 0.76 & 1.1 & 4.5 & 654 & 0.6 & 0.6 & 13.8 & 3.2 & 4.4\end{array}$ $\begin{array}{llllllllllllll}4.0 & 1.5 & 2.6 & \mathrm{M} / \mathrm{AV} & 370 & 1.5 & 3.0 & \text { ???? } & 1.0 & 3.5\end{array}$

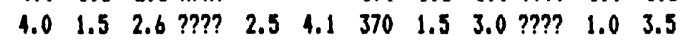
$\begin{array}{lllllllllllll}10.2 & 1.2 & 3.8 & 3.69 & 0.8 & 4.2 & 570 & 1.8 & 1.9 & 15.6 & 0.6 & 2.4\end{array}$ $\begin{array}{llllllllll}3.3 & 1.0 & 3.2 & \text { N/DA } & 360 & 1.2 & \text { N/DA } & 15.8 & 1.0 & 2.3\end{array}$ $\begin{array}{llllllllll}2.8 & 1.0 & 2.0 & 0.85 & 0.6 & 1.5 & 280 & 0.7 & 1.6 & \text { N/DA }\end{array}$

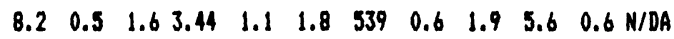
$\begin{array}{llllllllllll}4.6 & 0.9 & 1.1 & 1.79 & 1.0 & 1.2 & 625 & 1.0 & 1.4 & 8.3 & 1.3 & 1.2\end{array}$ N/DA N/DA N/DA $10.0 \mathrm{~N} / \mathrm{DA} \quad 1.5$

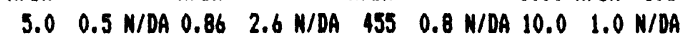

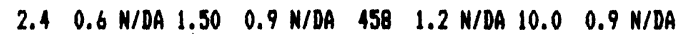
$2.40 .6 \mathrm{~N} / \mathrm{DA} \quad 1.50 \quad 0.9 \mathrm{~N} / \mathrm{DA} \quad 458 \quad 1.2 \mathrm{~N} / \mathrm{DA} \quad 10.0 \quad 1.0 \mathrm{~N} / \mathrm{DA}$

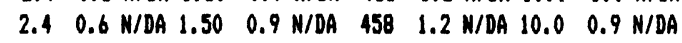
N/DA N/DA N/DA N/DA N/DA N/DA N/DA N/DA N/DA N/DA N/DA N/DA N/DA N/DA N/DA N/DA

N/DA N/DA N/DA N/DA

W/DA N/DA N/DA N/DA

N/DA N/DA N/DA N/DA

N/DA N/DA N/DA N/DA

N/AV N/AV N/AV 4.31 .21 .8

$\begin{array}{llllllllll}5.0 & 2.6 & \mathrm{~N} / \mathrm{DA} & 1.90 & 3.0 \mathrm{~N} / \mathrm{DA} & 510 & 1.9 \mathrm{~N} / \mathrm{DA} & 4.3 & 1.2 & 1.8\end{array}$

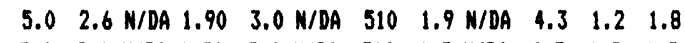

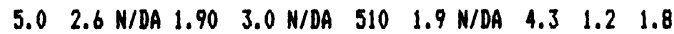

$\begin{array}{llllllllll}5.0 & 2.6 & \mathrm{~N} / \mathrm{DA} & 1.90 & 3.0 \mathrm{~N} / \mathrm{DA} & 510 & 1.9 \mathrm{~N} / \mathrm{DA} & 4.3 & 1.2 & 1.8\end{array}$

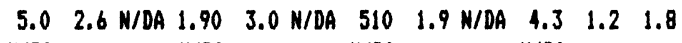

N/DA N/DA N/DA N/DA

N/DA N/DA N/DA N/DA

N/DA N/DA N/DA N/DA

N/DA N/DA N/DA N/DA

N/DA N/DA N/DA N/DA

$\begin{array}{llllll}2.2 & 1.1 & 1.4 \mathrm{~N} / \mathrm{AV} & 460 & 2.0 & 2.3 \mathrm{~N} / \mathrm{DA}\end{array}$

$\begin{array}{lllllllll}1.9 & 1.2 & 1.5 \mathrm{~N} / \mathrm{AV} & 440 & 2.1 & 2.2 & 10.9 & 0.5 \mathrm{~N} / \mathrm{DA}\end{array}$

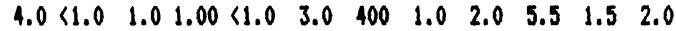

$\begin{array}{llllllllllllll}3.0 & 1.5 & 1.2 & 0.65 & 1.5 & 4.3 & 237 & 0.7 & 1.1 & 4.3 & 0.7 & 1.7\end{array}$

N/DA N/DA N/DA N/DA

W/DA N/DA N/DA N/DA

N/DA N/DA N/DA N/DA

$\begin{array}{llllllllllll}4.9 & 1.0 & 1.9 & 0.77 & 1.0 & 1.9 & 355 & 0.8 & 2.5 & 8.3 & 0.8 & 1.3\end{array}$

$\begin{array}{lllllllll}\text { N/DA } & \text { N/DA } & 247 & 3.0 & 4.3 & 5.1 & 2.0 & 3.4\end{array}$

$\begin{array}{llllllll}M / D A & \text { N/DA } & 247 & 3.0 & 4.3 & 5.1 & 2.0 & 3.4\end{array}$

$\begin{array}{llllllllllll}4.7 & 1.3 & 2.9 & 2.32 & 1.7 & 1.7 & 420 & 0.7 & 3.5 & 12.8 & 1.4 & 1.7\end{array}$

$\begin{array}{llllllllllll}4.7 & 1.3 & 2.9 & 2.32 & 1.7 & 1.7 & 420 & 0.7 & 3.5 & 12.8 & 1.4 & 1.7\end{array}$

$\begin{array}{llllllllllll}4.7 & 1.3 & 2.9 & 2.32 & 1.7 & 1.7 & 420 & 0.7 & 3.5 & 12.8 & 1.4 & 1.7\end{array}$

$\begin{array}{lllllllllllll}4.7 & 1.3 & 2.9 & 2.32 & 1.7 & 1.7 & 420 & 0.7 & 3.5 & 12.8 & 1.4 & 1.7\end{array}$

$\begin{array}{llllllllllll}4.3 & 0.9 & 1.7 & 0.95 & 2.8 & 5.6 & 226 & 1.6 & 2.4 & 11.1 & 1.8 & 2.7\end{array}$

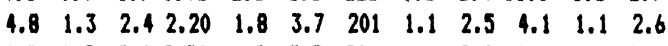

$\begin{array}{llllllllllll}4.8 & 1.3 & 2.4 & 2.20 & 1.8 & 3.7 & 201 & 1.1 & 2.5 & 4.1 & 1.1 & 2.6\end{array}$

$\begin{array}{llllllllllll}5.2 & 2.2 & 3.4 & 2.13 & 1.8 & 2.1 & 586 & 1.2 & 1.3 & 4.3 & 1.0 & 1.0\end{array}$

$\begin{array}{llllllllllll}3.2 & 1.6 & 3.0 & 2.66 & 0.9 & 1.6 & 274 & 1.3 & 2.0 & 5.1 & 1.2 & 1.3\end{array}$

$\begin{array}{llllllllllll}4.8 & 0.9 & 1.5 & 0.96 & 2.5 & 4.0 & 213 & 0.9 & 1.5 & 5.0 & 0.8 & 1.5\end{array}$

N/DA N/DA N/DA N/DA

M/AV N/AV N/AV N/AV

W/AV N/AV N/AV N/AV

M/AV N/AV N/AV N/AV

$\begin{array}{lllllllll}6.5 & 0.8 & 1.2 & 2.50 & 1.8 & 2.6 & 479 & 1.9 & 1.4\end{array} \mathrm{~N} / \mathrm{DA}$

$\begin{array}{llllllllll}6.4 & 0.9 & 1.2 & 2.49 & 2.2 & 3.4 & 400 & 1.3 & 2.0 & \mathrm{~N} / \mathrm{DA}\end{array}$

W/DA N/DA N/DA N/DA

N/DA N/DA N/DA N/DA

1.8 N/DA 1.93 .00 N/DA $1.2 \quad 150 \mathrm{~N} / \mathrm{DA} \quad 1.5 \mathrm{M} / \mathrm{DA}$

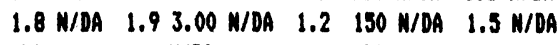

M/DA N/DA N/DA N/DA

N/DA N/DA N/DA N/DA

$3.8 \quad 1.2 \mathrm{~N} / \mathrm{DA} \mathrm{N} / \mathrm{DA} \quad 4002.1 \mathrm{~N} / \mathrm{DA}$ W/DA

$3.81 .2 \mathrm{~N} / D A \mathrm{~N} / \mathrm{DA} \quad 4002.1 \mathrm{~N} / \mathrm{DA}$ N/DA

W/DA N/DA N/DA N/DA

M/DA N/DA N/DA N/DA

N/DA N/DA N/DA N/DA 


\section{Acknowledgements}

This work would not have been possible without the generous help and co-operation of the companies manufacturing or marketing the analysers included in this review.

\section{References and evaluations}

Some of the instruments have been subject to DHSS and other evaluations. The following reports listed by instrument entry number in the data-base are available and can provide more detailed information.

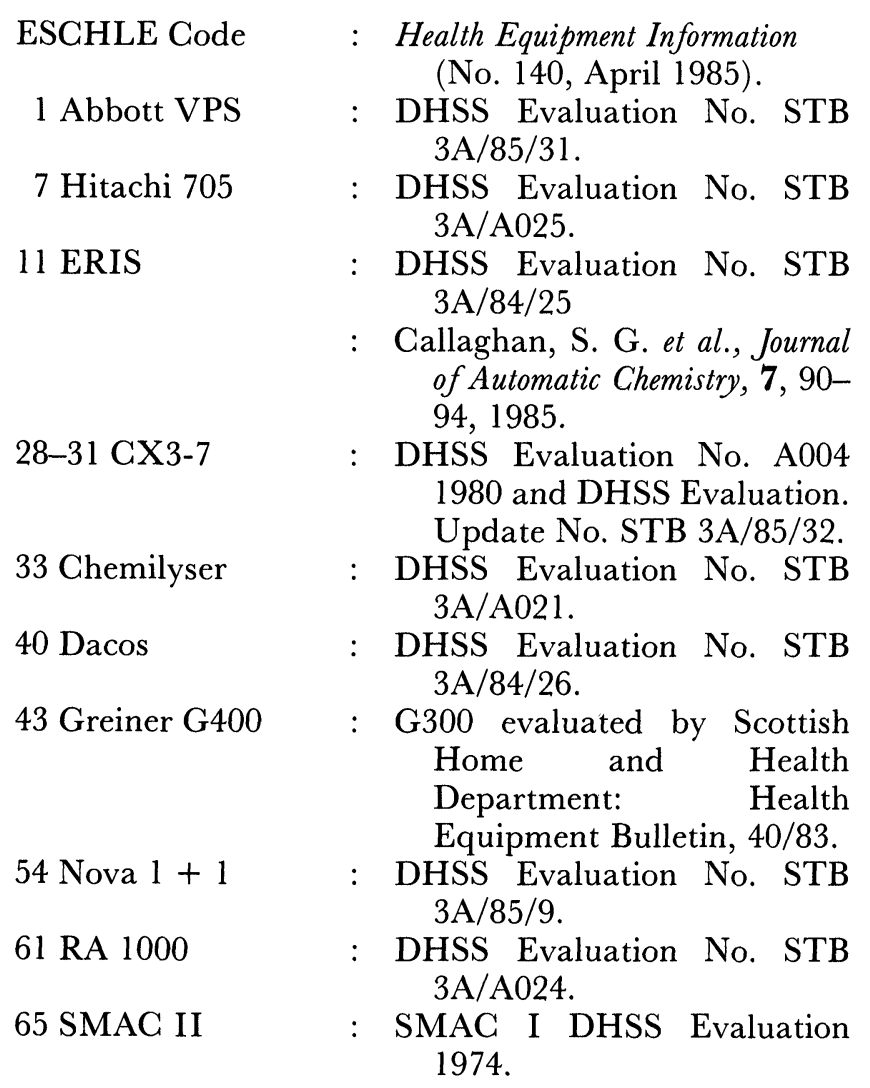

\section{Reference}

FyfFe, J. A., A review of large biochemistry analysers, 1985. Communications in Laboratory Medicine, I, 118-132.

\section{'CHEMICAL SENSORS CLUB NEWS'}

The eighth issue of CSC News (orders to J. A. Brown, 87 Gower Street, London WC1E 6AA) includes:

The pocket biosensor arrives; Analyticon: Gas and vapour sensors; Sensor research at Durham; Japanese sensor newsletter; Eurosensors 1987; Club database; Biotechnology Equipment Unit; School on Process Analysis.

\section{ANALYTICA 88}

To be held from 19 to 22 April 1988 in München, FR Germany

Analytica is a trade fair combined with an international conference - the conference this year being 'Biochemical Analytics'.

About a third of the companies participating in ANALYTICA - about 600 firms are expected to register - come from outside FR Germany. It is anticipated that the largest numbers of exhibitors from abroad will be from the UK (37 companies), the USA (31), Switzerland (18), France (9) and Italy (10).

In addition to individual exhibitors, France, the UK, Israel and the USA will also have official joint presentations. The UK's joint presentation includes 18 exhibitors; the exact numbers of exhibitors in the presentations of France, Israel and USA are not yet known.

\section{The Conference}

The scientific programme of the 11 th International Conference on Biochemical Analysis, 'Biochemical Analytics 88' (chaired by Professor Dr Helmut Greiling, Aachen), will again consist of three parts: symposia, poster exhibition and ANALYTICA Forum Munich.

In 1986 the conference attracted some 1250 participants. The number of visitors is expected to be far higher in 1988. Some 3200 professionals from 40 countries have expressed an interest in the conference.

The symposia will deal with the latest analytical developments both from the point of view of methodology and of the various fields of application. Special attention will be paid to problems of analytics, such as water analytics, including environmental biotechnology, as well as modern methods of molecular biology and gene technology.

In addition to the trade fair, the ANALYTICA Forum Munich provides the exhibiting industry with the opportunity of giving lectures in which companies can present and discuss their latest developments in industrial instrumentation and methodology.

For further information contact: Münchener Messe- und Ausstellungsgesellschaft mbH, Postfach 1210 09, D-8000 Munich 12, FR Germany. Tel.: (89) 5107-0, Telex 5212086 ameg $d$. 


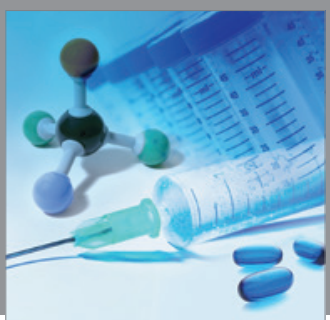

International Journal of

Medicinal Chemistry

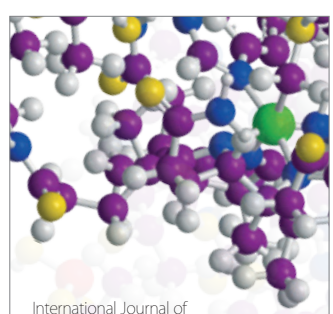

Carbohydrate Chemistry

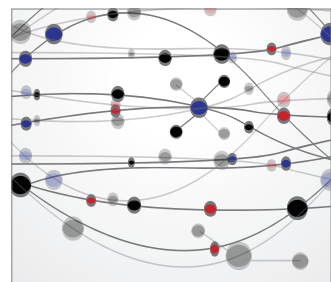

The Scientific World Journal
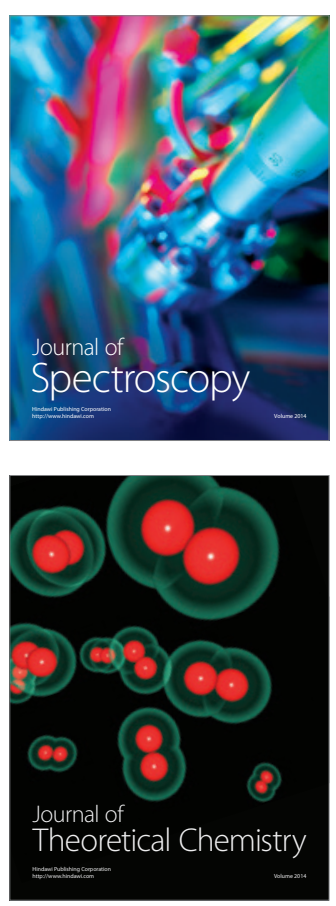
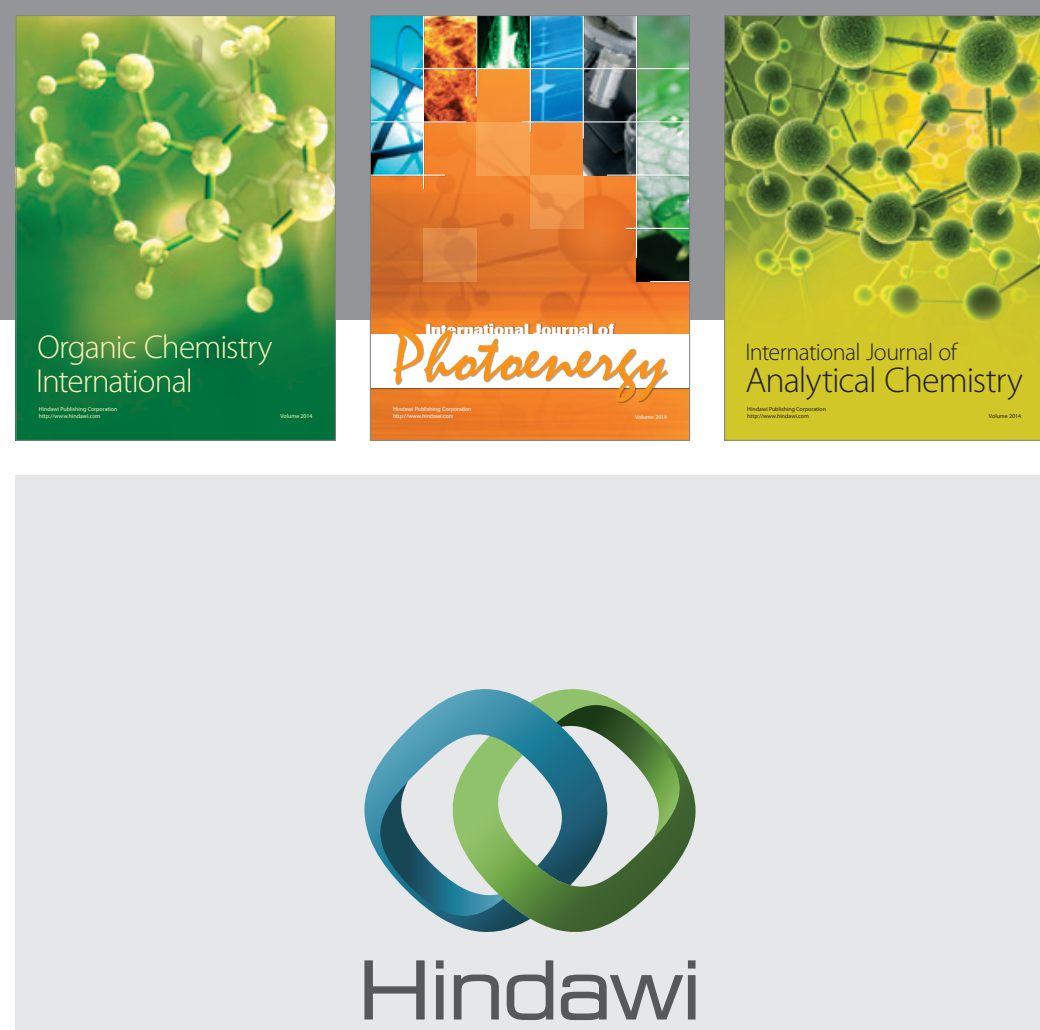

Submit your manuscripts at

http://www.hindawi.com
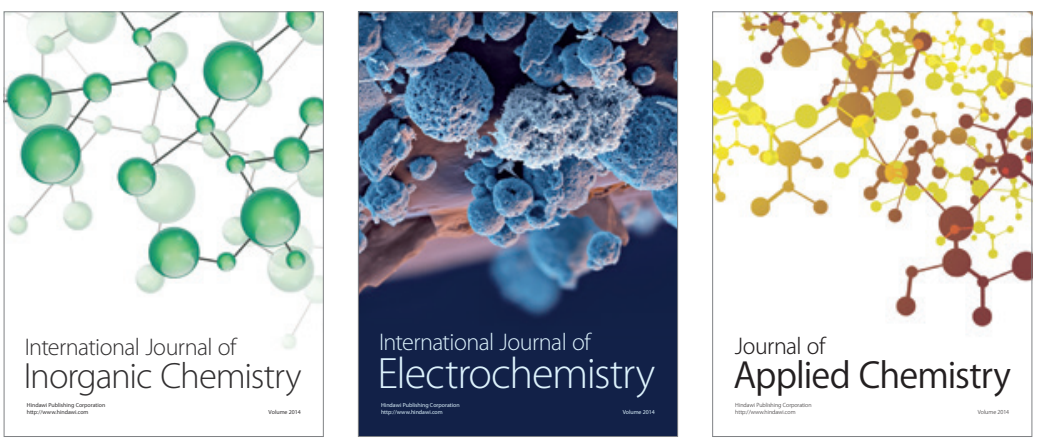

Journal of

Applied Chemistry
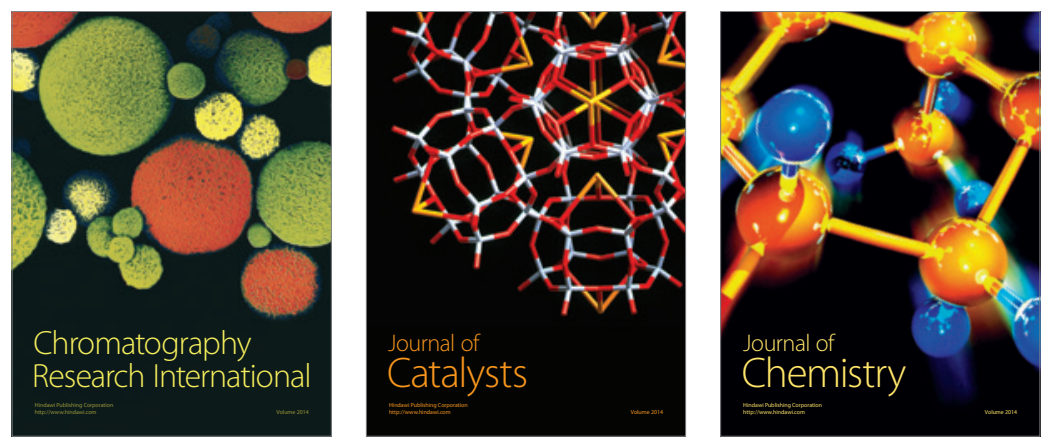
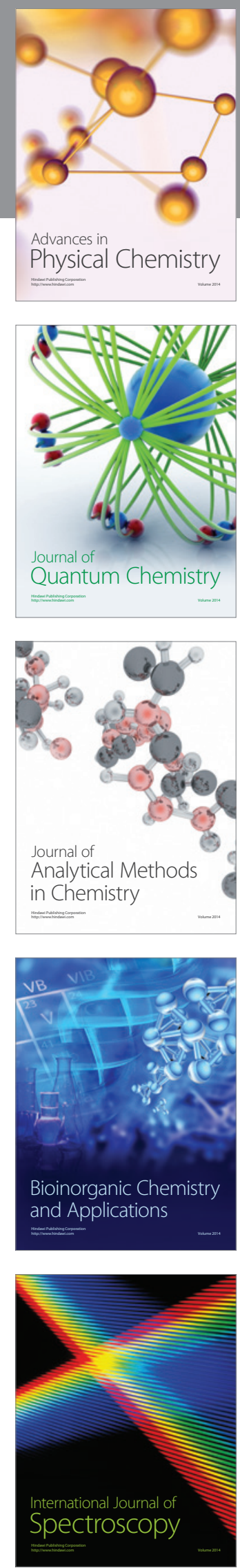